
Revue canadienne de chimie

\title{
Octahedral Co(III) Salen Complexes: The Role of Peripheral Ligand Electronics on Axial Ligand Release upon Reduction
}

\begin{tabular}{|c|c|}
\hline Journal: & Canadian Journal of Chemistry \\
\hline Manuscript ID & cjc-2017-0277.R1 \\
\hline Manuscript Type: & Article \\
\hline Date Submitted by the Author: & 06-Jun-2017 \\
\hline Complete List of Authors: & $\begin{array}{l}\text { Zhang, Chen; Simon Fraser University, Chemistry } \\
\text { Sutherland, Mathew; Simon Fraser University, Chemistry } \\
\text { Herasymchuk, Khrystyna; Simon Fraser University, Chemistry } \\
\text { Clarke, Ryan; Simon Fraser University, Chemistry } \\
\text { Thompson, John; Simon Fraser University, Chemistry } \\
\text { Chiang, Linus; Simon Fraser University, Chemistry } \\
\text { Walsby, Charles; Simon Fraser University } \\
\text { Storr, Tim; Simon Fraser University, Chemistry }\end{array}$ \\
\hline $\begin{array}{l}\text { Is the invited manuscript for } \\
\text { consideration in a Special } \\
\text { Issue? }\end{array}$ & SFU \\
\hline Keyword: & Cobalt, Hypoxia, Reduction, Ligand exchange, Cancer \\
\hline
\end{tabular}




\section{Octahedral Co(III) Salen Complexes: The Role of Peripheral Ligand Electronics on Axial Ligand Release upon Reduction}

Chen Zhang, ${ }^{\mathrm{a}}$ Mathew Sutherland, ${ }^{\mathrm{a}}$ Khrystyna Herasymchuk, ${ }^{\mathrm{a}}$ Ryan M. Clarke, ${ }^{\mathrm{a}} \mathrm{J}$ ohn R. Thompson, ${ }^{a}$ Linus Chiang, ${ }^{\text {a, }}$ Charles J. Walsby, ${ }^{\text {a* }}$ and Tim Storr ${ }^{\mathrm{a} *}$

${ }^{\mathrm{a}}$ Department of Chemistry, Simon Fraser University, Burnaby, BC, Canada

${ }^{\mathrm{b}}$ Present address: Department of Chemistry, University of the Fraser Valley, Abbotsford, BC, Canada

Emails: cwalsby@sfu.ca, tim_storr@,sfu.ca

Key Words: Cobalt, Cancer, Hypoxia, Reduction, Ligand Exchange 


\begin{abstract}
:
A series of octahedral Co ${ }^{\mathrm{III}}$ salen complexes (where salen represents a $\mathrm{N}_{2} \mathrm{O}_{2}$ bis-Schiff-base bisphenolate framework) were prepared with axial imidazole ligating groups. When using 1methylimidazole (1-MeIm) axial ligands, the $\mathrm{Co}^{\mathrm{III}} / \mathrm{Co}^{\mathrm{II}}$ reduction potential could be altered by $220 \mathrm{mV}$ via variation of the electron-donating ability of the para-ring substituents $(\mathrm{R}=\mathrm{H}(\mathbf{1})$, OMe (2), $\mathrm{tBu}(3), \mathrm{Br}(4), \mathrm{NO}_{2}(\mathbf{5})$, and $\left.\mathrm{CF}_{3}(\mathbf{6})\right)$. In addition, the irreversibility of the reduction process suggested substantial geometrical changes and axial ligand exchange upon reduction to the more labile $\mathrm{Co}^{\mathrm{II}}$ oxidation state. Installing an imidazole-coumarin conjugate as the axial ligands resulted in fluorescence quenching when bound to the $\mathrm{Co}^{\mathrm{III}}$ centre $(\mathrm{R}=\mathrm{H}(\mathbf{7}), \mathrm{OMe}(\mathbf{8})$, and $\mathrm{CF}_{3}(\mathbf{9})$ ). The redox properties and fluorescence increase upon ligand release for 7-9 were studied under reducing conditions, and in the presence of excess competing ligand (1-MeIm). It was determined that the Lewis acidity of the $\mathrm{Co}^{\mathrm{III}}$ centre was the dominant factor in controlling axial ligand exchange for this series of complexes.
\end{abstract}




\section{Introduction:}

There is an increasing interest in metal-based drugs for diagnostic and therapeutic applications, due to the need to increase both potency and selectivity. ${ }^{1-5}$ The ability to vary the coordination number, geometry, redox states, and overall charge provide major design advantages to metal-based over organic-based drugs. In addition, the ligand(s) play a key role in determining the pharmacokinetic properties of metal-containing drugs, allowing for tuning of the complex to increase efficacy. ${ }^{6}$ Metal complexes have been extensively studied as anticancer agents, with the development of Cisplatin playing an instrumental role in bringing medicinal inorganic chemistry into the mainstream. ${ }^{7}$ In certain cases, the ligand(s) can also be active drugs, and complexation to a metal complex can provide a means for selective delivery and/or activation. ${ }^{8}$ Metal complexes are well-suited as tumour-selective drug delivery agents, and can be activated by environmental factors such as $\mathrm{pH}^{9-10}$ redox, ${ }^{11}$ and light. ${ }^{12-13}$ The work herein focuses on the utilization of redox changes for metal complex activation.

A number of transition metal ions exhibit changes in geometrical preference and/or lability in different oxidations states, and these features have been exploited in the design of metal complexes that undergo selective activation in environments with different redox states. In the case of cancer, solid tumours exhibit a reduced $\mathrm{O}_{2}$ supply, which in combination with elevated levels of reducing agents and reductase enzymes results in a hypoxic microenvironment. ${ }^{14-16}$ A number of metal-based pro-drugs have been developed to target hypoxic tumour tissues, including complexes of $\mathrm{Pt}^{\mathrm{IV}}, \mathrm{Ru}^{\mathrm{III}}$, and $\mathrm{Co}^{\mathrm{III}}$ (Figure 1). ${ }^{8,11,17}$ Reduction of the $\mathrm{Pt}^{\mathrm{IV}}$ complex Satraplatin (Figure 1A) produces the activated $\mathrm{Pt}^{\mathrm{II}}$ species. Reduction of the $\mathrm{Ru}^{\mathrm{III}}$ complex NKP1339 (Figure 1B) ${ }^{18}$ to $\mathrm{Ru}^{\mathrm{II}}$ is hypothesized as one mode of activation for this important class of anticancer agents, ${ }^{19}$ however, recent studies suggest that the $\mathrm{Ru}{ }^{\mathrm{III}}$ oxidation 
state may be predominant in vivo. ${ }^{20-21}$ Both Satraplatin and NKP1339 have entered clinical trials. The Co ${ }^{\mathrm{III}}$ complex of a nitrogen mustard (Figure 1C) exhibited a 20-fold increase in cytotoxicity under hypoxic conditions, ${ }^{22} \mathrm{Co}^{\mathrm{III}}$ is kinetically inert whereas $\mathrm{Co}^{\mathrm{II}}$ is quite labile and undergoes rapid ligand exchange with water. ${ }^{23}$

Figure 1. Representative redox-activated anticancer drugs including (A) the $\mathrm{Pt}^{\mathrm{IV}}$ complex Satraplatin, (B) the $\mathrm{Ru}^{\mathrm{III}}$ complex NKP1339, (C) a Co ${ }^{\mathrm{III}}$-nitrogen mustard conjugate, and (D) $\mathrm{Co}^{\mathrm{III}}$ salen complexes investigated in this work, where $\mathrm{R}=\mathrm{H}, \mathrm{OMe}, \mathrm{tBu}, \mathrm{Br}, \mathrm{NO}_{2}$, or $\mathrm{CF}_{3}$, and $\mathrm{L}$ are imidazole ligands.

Bioreductive $\mathrm{Co}^{\mathrm{III}}$ pro-drugs of a number of antitumour agents have been developed, including nitrogen mustards, ${ }^{22}$ matrix metalloproteinase inhibitors, ${ }^{24}$ and curcumin. ${ }^{25}$ A key feature of these complexes, and other bioreductive pro-drugs, is the ability to tune the reduction potential so that activation occurs selectively under hypoxic conditions in a tumour. ${ }^{26}$ The approximate cellular reduction potential is estimated to be between -420 to $-150 \mathrm{mV}$ (vs. NHE), ${ }^{27}$ and the reduction potential of a tumour is usually at the lower end of this range. ${ }^{28}$ Previously, we showed that it is possible to tune the reduction potential of metal salen (salen represents an $\mathrm{N}_{2} \mathrm{O}_{2}$ bis-Schiff-base bis-phenolate framework., Figure 1D) complexes over a large range via alteration of the para-ring substituents, ${ }^{29-32}$ and we have now extended this work to a series of octahedral $\mathrm{Co}^{\mathrm{III}}$ salen complexes (Figure 1D). It was hypothesized that bioreduction to $\mathrm{Co}^{\mathrm{II}}$ would lead to significant geometrical rearrangement and axial ligand exchange (loss of L in Figure 1D), thus providing a selective release mechanism for cytotoxic ligands in the axial position. In addition, a number of reports have demonstrated the cytotoxicity of $\mathrm{Co}^{\mathrm{II}}$ salen complexes, a product of the reduction process. ${ }^{33-34}$ Herein, we detail the synthesis and electrochemical properties of a series of octahedral $\mathrm{Co}^{\mathrm{III}}$ salen complexes, and preliminary 
studies on axial ligand exchange under reducing conditions.

\section{Experimental:}

2.1 General Considerations: All reagents and solvents used for synthesis were from commercial sources and used as received. Literature methods were used or adapted to prepare the salen $\left(\mathrm{H}_{2} \mathrm{Sal}^{\mathrm{R}}\right)$ ligands $\left(\mathrm{R}=\mathrm{H}, \mathrm{OMe},{ }^{t} \mathrm{Bu}, \mathrm{H}, \mathrm{Br}, \mathrm{CF}_{3}, \mathrm{NO}_{2}\right)^{31,35-36}$ and $\mathrm{Co}^{\mathrm{II}}\left(\mathrm{Sal}^{\mathrm{R}}\right)$ complexes $(\mathrm{R}=\mathrm{H}$, OMe, $\left.{ }^{t} \mathrm{Bu}, \mathrm{Br}, \mathrm{CF}_{3}, \mathrm{NO}_{2}\right) .{ }^{31}$ 4-((1H-imidazol-1-yl) methyl)-7-methoxy-2H-chromen-2-one (hereafter named Coumarin) was prepared according to a literature method. ${ }^{37}$ Perchlorate salts are potentially explosive and in this work only small quantities were used in all syntheses. ${ }^{1} \mathrm{H}$ NMR spectra were recorded on Bruker AV-400 or AV-500 instruments. Mass spectra (positive ion) were obtained on an Agilent 6210 TOF ESI-MS instrument. Elemental analyses (C, H, N) were performed by Mr. Paul Mulyk at Simon Fraser University on a Carlo Erba EA1110 CHN elemental analyzer. Cyclic voltammetry (CV) was performed on a PAR-263A potentiometer, equipped with a glassy carbon working electrode and a platinum counter electrode, and a BASi MF-2062 non-aqueous electrode kit was used to make a silver/silver nitrate reference electrode with ${ }^{\mathrm{n}} \mathrm{Bu}_{4} \mathrm{NClO}_{4}(0.1 \mathrm{M})$ solutions in DMF. Ferrocene was used as an internal standard. Absorption spectra were collected using a Varian CARY 5000 UV-VisibleNear IR spectrophotometer. Measurements were performed in single-beam mode with baseline correction using the same quartz cuvette $(1 \mathrm{~cm}$ path length) in all experiments. The excitation and emission spectra were collected using a Photon Technology International (PTI) 814 photomultiplier detection system. Signals were collected at $90^{\circ}$ to the incident excitation beam. The maximum emission of the Coumarin solution was used as a reference. The photophysical data were processed with the Felix32 system. The kinetic studies of Coumarin ligand release 
over $12 \mathrm{~h}$ were performed at $25^{\circ} \mathrm{C}$ under constant agitation, and each well in the 96 -well plate was excited at $327 \mathrm{~nm}$ and emission was collected at $400 \mathrm{~nm}$ using a Synergy 4 fluorescence plate reader from BioTek. Each condition was measured in triplicate.

\subsection{Synthesis:}

$\left[\mathrm{Co}^{\mathrm{III}}\left(\mathrm{Sal}^{\mathrm{H}}\right)\left(1-\mathrm{MeIm}_{2}\right] \mathrm{ClO}_{4}(\mathbf{1}): \mathrm{To}^{\mathrm{Co}}\left(\mathrm{Sal}^{\mathrm{H}}\right)(0.1 \mathrm{~g}, 0.3 \mathrm{mmol})\right.$ in a $\mathrm{MeOH} / \mathrm{DCM}$ solution $(25 / 25 \mathrm{~mL})$ was added 3 eq. of 1 -methylimidazole (1-MeIm) $(0.076 \mathrm{~g}, 0.920 \mathrm{mmol})$. The solution was stirred at $298 \mathrm{~K}$ for $2 \mathrm{~h}$ and turned a dark red colour. $\mathrm{NaClO}_{4}(0.23 \mathrm{~g}, 1.80 \mathrm{mmol})$ in $\mathrm{MeOH}$ ( $2 \mathrm{~mL}$ ) was then added, and stirring in the air at $298 \mathrm{~K}$ was continued for 1 day. Slow evaporation of the solvent afforded dark red crystals after 1 day that were collected by filtration, washed with cold $\mathrm{MeOH}$, and dried in vacuo. Yield: $0.14 \mathrm{~g}, 79 \% .{ }^{1} \mathrm{H}$ NMR (400 MHz, MeOD) $\delta$ $8.20(\mathrm{~s}, 2 \mathrm{H}), 7.46(\mathrm{~s}, 2 \mathrm{H}), 7.32-7.25(\mathrm{~m}, 4 \mathrm{H}), 7.18(\mathrm{~d}, J=8.3 \mathrm{~Hz}, 2 \mathrm{H}), 6.94(\mathrm{t}, J=1.6 \mathrm{~Hz}, 2 \mathrm{H})$, $6.69(\mathrm{t}, J=1.5 \mathrm{~Hz}, 2 \mathrm{H}), 6.63-6.57(\mathrm{~m}, 2 \mathrm{H}), 4.06(\mathrm{~s}, 4 \mathrm{H}), 3.60(\mathrm{~s}, 6 \mathrm{H}) .{ }^{13} \mathrm{C} \mathrm{NMR}(101 \mathrm{MHz}$, $\mathrm{MeOD}) \delta 168.71,164.95,138.94,135.24,134.51,127.65,122.77,118.35,115.21,56.97,33.61$. $\operatorname{ESI}(+)-\mathrm{MS} \mathrm{m} / \mathrm{z}$ (relative intensity) $=489.15\left(\left[\mathrm{Co}^{\mathrm{III}}\left(\mathrm{Sal}^{\mathrm{H}}\right)(1-\mathrm{MeIm})_{2}\right]^{+}\right)$. Elemental analysis calculated for $\left[\mathrm{Co}^{\mathrm{III}}\left(\mathrm{Sal}^{\mathrm{H}}\right)(1-\mathrm{MeIm})_{2}\right] \mathrm{ClO}_{4}\left(\mathrm{C}_{24} \mathrm{H}_{26} \mathrm{~N}_{6} \mathrm{ClO}_{6} \mathrm{Co}\right) \mathrm{C}: 48.95 \%, \mathrm{H}: 4.45 \%, \mathrm{~N}: 14.27 \%$, Found C: $48.71 \%, \mathrm{H}: 4.65 \%, \mathrm{~N}: 14.11 \%$.

$\left[\mathrm{Co}^{\mathrm{III}}\left(\mathrm{Sal}^{\mathrm{OMe}}\right)(1-\mathrm{MeIm})_{2}\right] \mathrm{ClO}_{4}(2)$ : This complex was synthesized following the same procedure as for compound 1, combining $\mathrm{Co}^{\mathrm{II}}\left(\mathrm{Sal}^{\mathrm{OMe}}\right)(0.100 \mathrm{~g}, 0.260 \mathrm{mmol}), 1-\mathrm{MeIm}(0.064 \mathrm{~g}, 0.780$ mmol) and $\mathrm{NaClO}_{4}(0.19 \mathrm{~g}, 1.56 \mathrm{mmol})$. X-ray quality crystals were isolated by slow evaporation of a MeOH solution. Yield: $0.163 \mathrm{~g}, 97 \% .{ }^{1} \mathrm{H}$ NMR (400 MHz, DMSO-d $\left.{ }_{6}\right) \delta 8.18(\mathrm{~s}$, 2H), $7.54(\mathrm{t}, J=1.5 \mathrm{~Hz}, 2 \mathrm{H}), 7.08(\mathrm{t}, J=1.6 \mathrm{~Hz}, 2 \mathrm{H}), 6.99(\mathrm{~d}, J=9.1 \mathrm{~Hz}, 2 \mathrm{H}), 6.93(\mathrm{dd}, J=9.2$, 
$3.1 \mathrm{~Hz}, 2 \mathrm{H}), 6.79(\mathrm{~d}, J=3.1 \mathrm{~Hz}, 2 \mathrm{H}), 6.64(\mathrm{t}, J=1.5 \mathrm{~Hz}, 2 \mathrm{H}), 4.00(\mathrm{~s}, 4 \mathrm{H}), 3.65(\mathrm{~s}, 6 \mathrm{H}), 3.57(\mathrm{~s}$, $6 \mathrm{H}) .{ }^{13} \mathrm{C}$ NMR $\left(101 \mathrm{MHz}, \mathrm{DMSO}-d_{6}\right) \delta 167.90,160.42,148.20,138.67,127.42,124.81,123.02$, $121.97,116.30,114.66,56.85,55.45,34.47$. Elemental analysis calculated for $\left[\mathrm{Co}^{\mathrm{III}}\left(\mathrm{Sal}^{\mathrm{OMe}}\right)(1-\right.$ MeIm $\left.)_{2}\right] \mathrm{ClO}_{4}\left(\mathrm{C}_{26} \mathrm{H}_{30} \mathrm{~N}_{6} \mathrm{ClO}_{8} \mathrm{Co}\right) \mathrm{C}: 48.12 \%, \mathrm{H}: 4.66 \%, \mathrm{~N}: 12.95 \%$, Found $\mathrm{C}: 48.30 \%, \mathrm{H}$ : $4.96 \%, \mathrm{~N}: 12.76 \%$.

$\left[\mathrm{Co}^{\mathrm{III}}\left(\mathrm{Sal}^{t \mathrm{Bu}}\right)(1-\mathrm{MeIm})_{2}\right] \mathrm{ClO}_{4}(3)$ : This complex was synthesized following the same procedure as for compound 1, combining $\mathrm{Co}^{\mathrm{II}}\left(\mathrm{Sal}^{\mathrm{tBu}}\right)(0.063 \mathrm{~g}, 0.145 \mathrm{mmol}), 1-\mathrm{MeIm}(0.036 \mathrm{~g}$, $0.435 \mathrm{mmol})$ and $\mathrm{NaClO}_{4}(0.100 \mathrm{~g}, 0.869 \mathrm{mmol})$. Yield: $0.06 \mathrm{~g}, 90 \% .{ }^{1} \mathrm{H} \mathrm{NMR}(500 \mathrm{MHz}$, DMSO-d $\left.d_{6}\right) \delta 8.21(\mathrm{~s}, 2 \mathrm{H}), 7.57(\mathrm{~d}, J=1.5 \mathrm{~Hz}, 2 \mathrm{H}), 7.30(\mathrm{dd}, J=8.9,2.7 \mathrm{~Hz}, 2 \mathrm{H}), 7.19(\mathrm{~d}, J=$ $2.7 \mathrm{~Hz}, 2 \mathrm{H}), 7.07(\mathrm{t}, J=1.6 \mathrm{~Hz}, 2 \mathrm{H}), 7.01(\mathrm{~d}, J=8.9 \mathrm{~Hz}, 2 \mathrm{H}), 6.66(\mathrm{t}, J=1.5 \mathrm{~Hz}, 2 \mathrm{H}), 4.04(\mathrm{~s}$, 4H), $3.57(\mathrm{~s}, 6 \mathrm{H}), 1.21(\mathrm{~s}, 18 \mathrm{H}) .{ }^{13} \mathrm{C}$ NMR (101 MHz, DMSO-d $) \delta 168.60,163.44,138.63$, $135.75,132.94,129.93,127.42,122.04,121.98,116.66,56.73,34.51,33.29,31.14 . \mathrm{ESI}(+)-\mathrm{MS}$ $\mathrm{m} / \mathrm{z}$ (relative intensity) $=601.27\left(\left[\mathrm{Co}^{\mathrm{III}}\left(\mathrm{Sal}^{t \mathrm{Bu}}\right)(1-\mathrm{MeIm})_{2}\right]^{+}\right)$. Elemental analysis calculated for $\left[\mathrm{Co}^{\mathrm{III}}\left(\mathrm{Sal}^{t \mathrm{Bu}}\right)(1-\mathrm{MeIm})_{2}\right] \mathrm{ClO}_{4} \cdot 2 \mathrm{H}_{2} \mathrm{O} \cdot 2 \mathrm{CH}_{2} \mathrm{Cl}_{2} \quad\left(\mathrm{C}_{34} \mathrm{H}_{50} \mathrm{~N}_{6} \mathrm{Cl}_{5} \mathrm{O}_{8} \mathrm{Co}\right) \quad \mathrm{C}: \quad 45.02 \%, \mathrm{H}: \quad 5.56 \%, \mathrm{~N}:$ 9.27\%, Found C: $44.43 \%$, H: 5.32\%, N: 9.74\%.

$\left[\mathrm{Co}^{\mathrm{III}}\left(\mathrm{Sal}^{\mathrm{Br}}\right)(1-\mathrm{MeIm})_{2}\right] \mathrm{ClO}_{4}(4)$ : This complex was synthesized following the same procedure as for compound 1, combining $\mathrm{Co}^{\mathrm{II}}\left(\mathrm{Sal}^{\mathrm{Br}}\right)(0.100 \mathrm{~g}, 0.207 \mathrm{mmol}), 1-\mathrm{MeIm}(0.050 \mathrm{~g}, 0.621 \mathrm{mmol})$ and $\mathrm{NaClO}_{4}(0.15 \mathrm{~g}, 1.24 \mathrm{mmol})$. Yield: $0.12 \mathrm{~g}, 78 \% .{ }^{1} \mathrm{H}$ NMR $\left(500 \mathrm{MHz}, \mathrm{DMSO}-d_{6}\right) \delta 8.19$ (s, 2H), $7.56(\mathrm{~s}, 2 \mathrm{H}), 7.46(\mathrm{~d}, J=2.7 \mathrm{~Hz}, 2 \mathrm{H}), 7.27(\mathrm{dd}, J=9.1,2.7 \mathrm{~Hz}, 2 \mathrm{H}), 7.09(\mathrm{~d}, J=1.6 \mathrm{~Hz}$, 2H), $6.98(\mathrm{~d}, J=9.1 \mathrm{~Hz}, 2 \mathrm{H}), 6.61(\mathrm{~d}, J=1.5 \mathrm{~Hz}, 2 \mathrm{H}), 3.99(\mathrm{~s}, 4 \mathrm{H}), 3.56(\mathrm{~s}, 6 \mathrm{H}) .{ }^{13} \mathrm{C}$ NMR $(101$ MHz, DMSO- $\left.d_{6}\right) \delta 168.05,164.37,138.86,137.02,135.83,127.31,124.57,122.30,119.64$, 
104.17, 57.06, 34.56. $\mathrm{ESI}(+)-\mathrm{MS} \mathrm{m} / \mathrm{z}$ (relative intensity) $=646.97\left(\left[\mathrm{Co}^{\mathrm{III}}\left(\mathrm{Sal}^{\mathrm{Br}}\right)(1-\mathrm{MeIm})_{2}\right]^{+}\right)$. $\left[\mathrm{Co}^{\mathrm{III}}\left(\mathrm{Sal}^{\mathrm{Br}}\right)\left(1-\mathrm{MeIm}_{2}\right] \mathrm{ClO}_{4} \bullet 0.5 \mathrm{CH}_{2} \mathrm{Cl}_{2} \quad\left(\mathrm{C}_{24.5} \mathrm{H}_{25} \mathrm{~N}_{6} \mathrm{Cl}_{2} \mathrm{O}_{6} \mathrm{CoBr}_{2}\right) \quad \mathrm{C}: 37.29 \%, \quad \mathrm{H}: \quad 3.19 \%, \mathrm{~N}:\right.$ 10.65\%, Found C: $37.16 \%, \mathrm{H}: 3.59 \%, \mathrm{~N}: 10.65 \%$.

$\left[\mathrm{Co}^{\mathrm{III}}\left(\mathrm{Sal}^{\mathrm{NO} 2}\right)(1-\mathrm{MeIm})_{2}\right] \mathrm{ClO}_{4}(\mathbf{5})$ : This complex was synthesized following the same procedure as for compound 1, combining $\mathrm{Co}^{\mathrm{II}}\left(\mathrm{Sal}^{\mathrm{NO} 2}\right)(0.100 \mathrm{~g}, 0.240 \mathrm{mmol}), 1-\mathrm{MeIm}(0.060 \mathrm{~g}, 0.722$ mmol) and $\mathrm{NaClO}_{4}(0.176 \mathrm{~g}, 1.440 \mathrm{mmol})$. Yield: $0.11 \mathrm{~g}, 79 \% .{ }^{1} \mathrm{H}$ NMR (400 MHz, DMSO- $\left.d_{6}\right)$ $\delta 8.48(\mathrm{~s}, 2 \mathrm{H}), 8.46(\mathrm{~d}, J=2.7 \mathrm{~Hz}, 2 \mathrm{H}), 8.07(\mathrm{dd}, J=9.3,2.8 \mathrm{~Hz}, 2 \mathrm{H}), 7.68(\mathrm{~s}, 2 \mathrm{H}), 7.23-7.14$ $(\mathrm{m}, 4 \mathrm{H}), 6.67(\mathrm{~s}, 2 \mathrm{H}), 4.12(\mathrm{~s}, 4 \mathrm{H}), 3.59(\mathrm{~s}, 6 \mathrm{H}) .{ }^{13} \mathrm{C}$ NMR $(101 \mathrm{MHz}$, DMSO-d $) \delta 170.58$, $169.56,139.14,135.62,132.54,128.91,127.12,122.89,122.77,117.59,57.41,34.67 . \mathrm{ESI}(+)-$ MS m/z (relative intensity) $=579.11\left(\left[\mathrm{Co}^{\mathrm{III}}\left(\operatorname{salen}^{\mathrm{NO} 2}\right)(1-\mathrm{MeIm})_{2}\right]^{+}\right)$. Elemental analysis calculated for $\left[\mathrm{Co}^{\mathrm{III}}\left(\mathrm{Sal}^{\mathrm{NO} 2}\right)(1-\mathrm{MeIm})_{2}\right] \mathrm{ClO}_{4}\left(\mathrm{C}_{24} \mathrm{H}_{24} \mathrm{~N}_{8} \mathrm{ClO}_{10} \mathrm{Co}\right) \mathrm{C}: 42.46 \%, \mathrm{H}: 3.56 \%$, N: 16.51\%, Found C: $42.87 \%$, H: 3.68\%, N: 16.04\%.

$\left[\mathrm{Co}^{\mathrm{III}}\left(\mathrm{Sal}^{\mathrm{CF} 3}\right)\left(1-\mathrm{MeIm}_{2}\right] \mathrm{ClO}_{4}(6)\right.$ : To a stirring solution of $\mathrm{H}_{2} \mathrm{Sal}^{\mathrm{CF} 3}(0.03 \mathrm{~g}, 0.0742 \mathrm{mmol})$ (in $\left.\mathrm{Et}_{2} \mathrm{O}\right)$ under $\mathrm{N}_{2}, \mathrm{Co}(\mathrm{OAc})_{2} \bullet 4 \mathrm{H}_{2} \mathrm{O}(0.018 \mathrm{~g}, 0.0742 \mathrm{mmol})$ in $\mathrm{MeOH}$ was added. The mixture was stirred for $20 \mathrm{~min}$ and then 3 eq. of $1-\operatorname{MeIm}(0.018 \mathrm{~g}, 0.223 \mathrm{mmol})$ and $\mathrm{NaClO}_{4}(0.176 \mathrm{~g}, 1.440$ mmol) were added to the reaction. The solution was then stirred under an aerobic atmosphere overnight and then concentrated under low pressure causing a precipitate to form. A dark red powder was collected by filtration, washed with cold methanol, and dried in vacuo. Yield: 0.04 g, 71\%. X-ray quality crystals were isolated by slow evaporation from MeOH. ${ }^{1} \mathrm{H}$ NMR (400 MHz, DMSO-d $)_{6} \delta 8.38(\mathrm{~s}, 2 \mathrm{H}), 7.75(\mathrm{~d}, J=2.4 \mathrm{~Hz}, 2 \mathrm{H}), 7.64(\mathrm{~s}, 2 \mathrm{H}), 7.48(\mathrm{dd}, J=9.1,2.6 \mathrm{~Hz}$, 2H), $7.19(\mathrm{~d}, J=8.9 \mathrm{~Hz}, 2 \mathrm{H}), 7.14(\mathrm{t}, J=1.6 \mathrm{~Hz}, 2 \mathrm{H}), 6.66(\mathrm{t}, J=1.6 \mathrm{~Hz}, 2 \mathrm{H}), 4.07(\mathrm{~s}, 4 \mathrm{H}), 3.59$ 
$(\mathrm{s}, 6 \mathrm{H}) .{ }^{13} \mathrm{C}$ NMR $\left(101 \mathrm{MHz}, \mathrm{DMSO}-d_{6}\right) \delta 169.47,168.19,139.45,130.93,127.73,126.50$, $123.69,122.98,118.05,115.24,114.91,57.62,35.08 .{ }^{19} \mathrm{~F} \mathrm{NMR}\left(500 \mathrm{MHz}, \mathrm{CDCl}_{3}\right) \delta-59.50(\mathrm{~s})$. $\mathrm{ESI}(+)-\mathrm{MS} \mathrm{m} / \mathrm{z}$ (relative intensity) $=625.14\left(\left[\mathrm{Co}^{\mathrm{III}}\left(\mathrm{Sal}^{\mathrm{CF} 3}\right)(1-\mathrm{MeIm})_{2}\right]^{+}\right)$. Elemental analysis calculated for $\left[\mathrm{Co}^{\mathrm{III}}\left(\mathrm{Sal}^{\mathrm{CF} 3}\right)(1-\mathrm{MeIm})_{2}\right] \mathrm{ClO}_{4} \cdot 2 \mathrm{H}_{2} \mathrm{O}\left(\mathrm{C}_{26} \mathrm{H}_{28} \mathrm{~N}_{6} \mathrm{ClO}_{8} \mathrm{Co}\right) \mathrm{C}: 41.04 \%, \mathrm{H}: 3.71 \%$, N: 11.04\%, Found C: 40.60\%, H: 3.37\%, N: 11.47\%.

$\left[\mathrm{Co}^{\mathrm{III}}\left(\mathrm{Sal}^{\mathrm{H}}\right)(\text { Coumarin })_{2}\right] \mathrm{Cl}(7)$ : Three drops of $\mathrm{NEt}_{3}$ was added to a solution of $\mathrm{H}_{2} \mathrm{Sal}^{\mathrm{H}}(0.123 \mathrm{~g}$, $0.378 \mathrm{mmol}$ in DCM $)$ in a round-bottom flask under $\mathrm{N}_{2} . \mathrm{CoCl}_{2}(0.050 \mathrm{~g}, 0.385 \mathrm{mmol}$ in $\mathrm{MeOH})$ was added into the flask, which was then purged with $\mathrm{N}_{2}$ for $20 \mathrm{~min}$. Then 4-((1H-imidazol-1-yl) methyl)-7-methoxy-2H-chromen-2-one (Coumarin $)^{37}(0.213 \mathrm{~g}, 0.831 \mathrm{mmol})$ was added to the reaction. The solution was stirred in air overnight. The solvent was then removed in vacuo, and a brown solid was obtained. The solid was washed with EtOAc and filtered, and then the crude product was further purified by size exclusion chromatography using a Sephadex ${ }^{\circledR}$ column (LH20; MeOH eluent). Yield: 0.28 g, 83\%. ${ }^{1} \mathrm{H}$ NMR (400 MHz, MeOD) $\delta 8.21(\mathrm{~s}, 1 \mathrm{H}), 7.74$ (t, $J=$ $1.5 \mathrm{~Hz}, 1 \mathrm{H}), 7.42(\mathrm{~d}, J=8.8 \mathrm{~Hz}, 1 \mathrm{H}), 7.27-7.18(\mathrm{~m}, 2 \mathrm{H}), 7.14-7.08(\mathrm{~m}, 2 \mathrm{H}), 6.85(\mathrm{~d}, J=2.5 \mathrm{~Hz}$, $1 \mathrm{H}), 6.83-6.78(\mathrm{~m}, 2 \mathrm{H}), 6.58-6.53(\mathrm{~m}, 1 \mathrm{H}), 5.40(\mathrm{~d}, J=1.4 \mathrm{~Hz}, 2 \mathrm{H}), 5.27(\mathrm{~d}, J=1.3 \mathrm{~Hz}, 1 \mathrm{H})$, $4.08(\mathrm{~s}, 2 \mathrm{H}), 3.85$ (s, 3H). ${ }^{13} \mathrm{C}$ NMR (101 MHz, MeOD) $\delta 170.42,166.26,164.89,162.26$, $156.74,151.99,141.09,136.96,135.86,129.65,125.99,123.83,122.94,119.86,117.03,113.94$, 110.73, 111.69, 111.40, 102.11, 58.37, 56.51. ESI (+)-MS m/z (relative intensity) $=837.21$ $\left(\left[\mathrm{Co}^{\mathrm{III}}\left(\mathrm{Sal}^{\mathrm{H}}\right)(\text { Coumarin })_{2}\right]^{+}\right) . \quad$ Elemental analysis for $\left[\mathrm{Co}^{\mathrm{III}}\left(\mathrm{Sal}^{\mathrm{H}}\right)(\text { Coumarin })_{2}\right] \mathrm{Cl} \cdot \mathrm{CH}_{2} \mathrm{Cl}_{2}\left(\mathrm{C}_{45} \mathrm{H}_{40} \mathrm{~N}_{6} \mathrm{Cl}_{3} \mathrm{O}_{8} \mathrm{Co}\right) \mathrm{C}: 56.41 \%$, H: $4.21 \%$, N: 8.77\%, Found C: $56.19 \%, H: 4.03 \%, \mathrm{~N}: 9.26 \%$. 
$\left[\mathrm{Co}^{\mathrm{III}}\left(\mathrm{Sal}^{\mathrm{OMe}}\right)(\mathrm{Coumarin})_{2}\right] \mathrm{Cl}(\mathbf{8})$ : The complex was synthesized following the same procedure as for 7, combining $\mathrm{H}_{2} \mathrm{Sal}^{\mathrm{OMe}}(0.13 \mathrm{~g}, 0.385 \mathrm{mmol}), \mathrm{CoCl}_{2}(0.05 \mathrm{~g}, 0.385 \mathrm{mmol})$ and Coumarin (0.22 g, $0.847 \mathrm{mmol})$. Yield: $0.35 \mathrm{~g}, 97 \% .{ }^{1} \mathrm{H}$ NMR (400 MHz, MeOD) $\delta 8.19$ (s, 1H), 7.73 (t, $J$ $=1.4 \mathrm{~Hz}, 1 \mathrm{H}), 7.45(\mathrm{~d}, J=9.0 \mathrm{~Hz}, 1 \mathrm{H}), 7.14(\mathrm{t}, J=1.7 \mathrm{~Hz}, 1 \mathrm{H}), 7.07(\mathrm{~d}, J=9.2 \mathrm{~Hz}, 1 \mathrm{H}), 6.96$ $(\mathrm{dd}, J=9.2,3.1 \mathrm{~Hz}, 1 \mathrm{H}), 6.91(\mathrm{~d}, J=2.5 \mathrm{~Hz}, 1 \mathrm{H}), 6.85(\mathrm{dd}, J=8.9,2.5 \mathrm{~Hz}, 1 \mathrm{H}), 6.82(\mathrm{t}, J=1.5$ $\mathrm{Hz}, 1 \mathrm{H}), 6.77$ (d, $J=3.2 \mathrm{~Hz}, 1 \mathrm{H}), 5.43(\mathrm{~d}, J=1.3 \mathrm{~Hz}, 2 \mathrm{H}), 5.38-5.34(\mathrm{~m}, 1 \mathrm{H}), 4.09$ (s, 2H), 3.89 (s, 3H), 3.73 (s, 3H). ${ }^{13} \mathrm{C}$ NMR (101 MHz, MeOD) $\delta 169.90,164.89,162.27,161.02,157.26$, $156.76,152.07,151.31,141.05,129.61,126.98,125.98,124.48,122.93,118.50,115.77,113.92$, 111.69, 110.86, 102.11, 58.35, 56.50, 56.27. ESI (+)-MS m/z (relative intensity) $=897.23$ $\left(\left[\mathrm{Co}^{\mathrm{III}}\left(\mathrm{Sal}^{\mathrm{OMe}}\right)(\mathrm{Coumarin})_{2}\right]^{+}\right) . \quad$ Elemental analysis for $\left[\mathrm{Co}^{\mathrm{III}}\left(\mathrm{Sal}^{\mathrm{OMe}}\right)(\text { Coumarin })_{2}\right] \mathrm{Cl} \bullet 1 \mathrm{CH}_{2} \mathrm{Cl}_{2} \bullet 0.5 \mathrm{H}_{2} \mathrm{O}\left(\mathrm{C}_{47} \mathrm{H}_{45} \mathrm{~N}_{6} \mathrm{Cl}_{3} \mathrm{O}_{10.5} \mathrm{Co}\right) \mathrm{C}: 54.96 \%, \mathrm{H}: 4.42 \%, \mathrm{~N}:$ $8.18 \%$, Found C: $54.82 \%$, H: $3.87 \%$, N: $8.88 \%$.

$\left[\mathrm{Co}^{\mathrm{III}}\left(\mathrm{Sal}^{\mathrm{CF} 3}\right)(\mathrm{Coumarin})_{2}\right] \mathrm{Cl}(9)$ : The complex was synthesized following the same procedure as for 7, combining $\mathrm{H}_{2} \mathrm{Sal}^{\mathrm{CF} 3}(0.071 \mathrm{~g}, 0.176 \mathrm{mmol}), \mathrm{CoCl}_{2}(0.023 \mathrm{~g}, 0.176 \mathrm{mmol})$ and Coumarin (0.090 g, $0.351 \mathrm{mmol})$. Yield: 94\%. ${ }^{1} \mathrm{H}$ NMR (400 MHz, MeOD) $\delta 8.37$ (s, 1H), $7.86(\mathrm{t}, J=1.4$ $\mathrm{Hz}, 1 \mathrm{H}), 7.71-7.67(\mathrm{~m}, 1 \mathrm{H}), 7.50(\mathrm{~d}, J=8.8 \mathrm{~Hz}, 2 \mathrm{H}), 7.27(\mathrm{~d}, J=9.0 \mathrm{~Hz}, 1 \mathrm{H}), 7.19(\mathrm{t}, J=1.7$ $\mathrm{Hz}, 1 \mathrm{H}), 6.91(\mathrm{~d}, J=2.5 \mathrm{~Hz}, 1 \mathrm{H}), 6.89-6.83(\mathrm{~m}, 2 \mathrm{H}), 5.47(\mathrm{~d}, J=1.4 \mathrm{~Hz}, 2 \mathrm{H}), 5.31(\mathrm{~s}, 1 \mathrm{H})$, $4.20(\mathrm{~s}, 2 \mathrm{H}), 3.89(\mathrm{~s}, 3 \mathrm{H}) .{ }^{19} \mathrm{~F}$ NMR (400 MHz, MeOD) $\delta-62.95$ (s). ${ }^{13} \mathrm{C}$ NMR (101 MHz, MeOD) $\delta 170.82,168.64,164.95,162.26,156.77,152.17,141.22,134.98,132.69,129.51$, $125.95,124.57,123.48,119.34,113.99,111.64,110.49,102.05,68.86,58.68,56.49,47.95,9.22$. ESI $(+)-\mathrm{MS} \mathrm{m} / \mathrm{z}$ (relative intensity) $=973.11\left(\left[\mathrm{Co}^{\mathrm{III}}\left(\mathrm{Sal}^{\mathrm{CF} 3}\right)(\text { Coumarin })_{2}\right]^{+}\right)$. Elemental analysis calculated for $\left[\mathrm{Co}^{\mathrm{III}}\left(\mathrm{Sal}^{\mathrm{CF} 3}\right)(\mathrm{Coumarin})_{2}\right] \mathrm{Cl} \bullet 0.5 \mathrm{CH}_{2} \mathrm{Cl}_{2} \bullet 3 \mathrm{H}_{2} \mathrm{O} \quad\left(\mathrm{C}_{46.5} \mathrm{H}_{43} \mathrm{~N}_{6} \mathrm{Cl}_{2} \mathrm{O}_{11} \mathrm{CoF}_{6}\right) \quad \mathrm{C}$ : 
50.51\%, H: 3.92\%, N: 7.60\%, Found C: 50.48\%, H: 4.53\%, N: 7.54\%.

2.3 X-ray Structure Determination: Single crystal X-ray crystallographic analyses of selected $\left[\mathrm{Co}^{\mathrm{III}}\left(\mathrm{Sal}^{\mathrm{R}}\right)(1-\mathrm{MeIm})_{2}\right] \mathrm{ClO}_{4}\left(\mathrm{R}=\mathrm{H}(\mathbf{1}), \mathrm{OMe}(\mathbf{2})\right.$, and $\left.\mathrm{CF}_{3}(\mathbf{6})\right)$ complexes were performed on a Bruker X8 APEX II diffractometer and each crystal was mounted on a $150 \mu \mathrm{m}$ MiTeGen glass fibre. For 1, data were collected to a maximum $2 \theta$ value of $57.2^{\circ}$, in a series of $\varphi$ and $\omega$ in $0.50^{\circ}$ widths with $10.0 \mathrm{~s}$ exposures. The crystal-to-detector distance was $50 \mathrm{~mm}$. For $\mathbf{2}$, data collection was completed with TRIUMPH graphite monochromated Mo K $\alpha$ radiation. Data were collected to a maximum $2 \theta$ value of $61.0^{\circ}$, in a series of $\varphi$ and $\omega$ in $0.50^{\circ}$ widths with $30.0 \mathrm{~s}$ exposures. The crystal-to-detector distance was $50 \mathrm{~mm}$. For $\mathbf{6}$, data collection was performed with graphite monochromated $\mathrm{Cu} \mathrm{K} \alpha$ radiation. Data were collected at $149 \pm 2 \mathrm{~K}$ to a maximum $2 \theta$ value of $133.3^{\circ}$, in a series of $\varphi$ and $\omega$ in $0.80^{\circ}$ widths with $10.0 \mathrm{~s}$ exposures. The crystal-to-detector distance was $40 \mathrm{~mm}$. The structures were solved by intrinsic phasing (SIR92) ${ }^{38}$ and refined by least-squares procedures using SHELXL-2013. ${ }^{39}$ Single-crystal X-ray crystallographic analysis of a red crystal of 9 was performed by the SCrALS (Service Crystallography at Advanced Light Source) program at the Small-Crystal Crystallography Beamline 11.3.1 at the Advanced Light Source (ALS), Lawrence Berkeley National Laboratory using synchrotron radiation tuned to $\lambda=$ $0.7749 \AA$ A. Intensity data were collected at $296 \mathrm{~K}$ on a D8 goniostat equipped with a Bruker APEXII CCD detector at Beamline 11.3.1. For data collection, frames were measured for a duration of $1 \mathrm{~s}$ at $0.3^{\circ}$ intervals of $\omega$ with a maximum $2 \theta$ value of $60^{\circ}$. The data frames were collected using the program APEX2 and processed using the program SAINT within APEX2. The data were corrected for absorption and beam corrections based on the multiscan technique as implemented in SADABS. The structure was solved by the intrinsic phasing method ${ }^{40}$ and 
subsequent refinements were performed using ShelXle. ${ }^{41}$ For all structures non-hydrogen atoms were refined anisotropically. All $\mathrm{C}-\mathrm{H}$ hydrogen atoms were placed in calculated positions but were not refined. A summary of the crystal data and experimental parameters for the structure determinations are given in Table 2.1. Crystallographic data has been deposited at CCDC with numbers $1545820-1545823$. All crystal structure plots were produced using ORTEP-3 ${ }^{42}$ and rendered with POV-Ray (v.3.6.2). ${ }^{43-44}$

Table 1. Selected crystallographic data for $\left[\mathrm{Co}^{\mathrm{III}}\left(\mathrm{Sal}^{\mathrm{R}}\right)(\mathrm{L})_{2}\right][\mathrm{X}]$ complexes.

\begin{tabular}{|c|c|c|c|c|}
\hline$\left[\mathrm{Co}^{\prime \prime \prime}\left(\mathrm{Sal}^{\mathrm{R}}\right)(\mathrm{L})_{2}\right] \mathrm{X}$ & 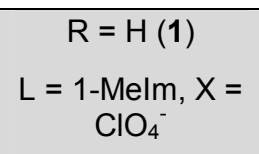 & 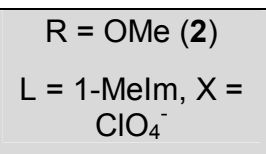 & $\begin{array}{c}\mathrm{R}=\mathrm{CF}_{3}(\mathbf{6}) \\
\mathrm{L}=\underset{1-\mathrm{MeIm}^{-}, \mathrm{X}=}{\mathrm{ClO}_{4}^{-}}\end{array}$ & $\begin{array}{c}\mathrm{R}=\mathrm{CF}_{3}(\mathbf{9}) \\
\mathrm{L}=\text { Coumarin, } \mathrm{X}=\mathrm{Cl}^{-}\end{array}$ \\
\hline Formula & $\mathrm{C}_{24} \mathrm{H}_{26} \mathrm{ClN}_{6} \mathrm{O}_{6} \mathrm{Co}$ & $\mathrm{C}_{26} \mathrm{H}_{30} \mathrm{ClN}_{6} \mathrm{O}_{8} \mathrm{Co}$ & $\mathrm{C}_{26} \mathrm{H}_{24} \mathrm{ClN}_{6} \mathrm{O}_{6} \mathrm{~F}_{6} \mathrm{Co}$ & $\mathrm{C}_{46} \mathrm{H}_{36} \mathrm{ClN}_{6} \mathrm{O}_{8} \mathrm{~F}_{6} \mathrm{Co}$ \\
\hline Formula weight & 588.89 & 648.94 & 724.89 & 1027.20 \\
\hline space group & P 212121 & $P-1$ & $P-1$ & $P-1$ \\
\hline$a(\AA)$ & $12.4129(7)$ & $8.4249(4)$ & $10.9036(5)$ & $10.5080(7)$ \\
\hline $\mathrm{b}(\AA \AA)$ & $13.1381(8)$ & $11.8610(6)$ & $11.0510(5)$ & $14.4981(10)$ \\
\hline$c(\AA)$ & $15.6039(9)$ & $15.0154(8)$ & $14.3468(7)$ & $16.3735(11)$ \\
\hline$\alpha\left({ }^{\circ}\right)$ & 90 & $74.3740(10)$ & $106.780(4)$ & $78.449(2)$ \\
\hline$\beta\left({ }^{\circ}\right)$ & 90 & $81.6870(10)$ & $96.598(3)$ & $87.379(2)$ \\
\hline $\mathrm{Y}\left({ }^{\circ}\right)$ & 90 & $76.8910(10)$ & $105.318(3)$ & $69.154(2)$ \\
\hline $\mathrm{V}\left[\AA^{3}\right]$ & $2544.7(3)$ & $1401.76(12)$ & 1561.74 & $2283.1(3)$ \\
\hline$Z, D_{\text {calc }}\left[\mathrm{g} / \mathrm{cm}^{3}\right]$ & 4 & 2 & 2 & 2 \\
\hline $\mathrm{T}(\mathrm{K})$ & $296(2)$ & $150(2)$ & $149(2)$ & $273(2)$ \\
\hline$\rho_{\text {calcd }}\left(\mathrm{g} \mathrm{cm}^{-3}\right)$ & 1.537 & 1.537 & 1.542 & 1.494 \\
\hline$\lambda(\AA)$ & 0.71073 & 0.71073 & 1.54178 & 0.7749 \\
\hline$\mu\left(\mathrm{cm}^{-1}\right)$ & 0.832 & 0.768 & 5.921 & 0.654 \\
\hline $\begin{array}{c}R \text { indices }{ }^{a} \text { with } \mid>2 \sigma(I) \\
\text { (data) }\end{array}$ & 0.0545 & 0.0285 & 0.0733 & 0.0903 \\
\hline $\mathrm{wR}_{2}$ & 0.1477 & 0.0731 & 0.1904 & 0.2629 \\
\hline $\mathrm{R}_{1}$ & 0.0660 & 0.0373 & 0.0879 & 0.1041 \\
\hline Goodness-of-fits on $\mathrm{F}^{2}$ & 1.027 & 1.028 & 1.063 & 1.024 \\
\hline
\end{tabular}




\section{Results and Discussion:}

3.1 Synthesis and Characterization of $\left[\mathrm{Co}^{\mathrm{III}}\left(\mathrm{Sal}^{\mathrm{R}}\right)(1-\mathrm{MeIm})_{2}\right]\left[\mathrm{ClO}_{4}\right]$ (1-6): We initially chose to target the synthesis of a series of octahedral $\left[\mathrm{Co}^{\mathrm{III}}\left(\mathrm{Sal}^{\mathrm{R}}\right)(\mathrm{L})_{2}\right]^{+}$complexes with neutral axial ligands, and 1-methylimidazole (1-MeIm) was chosen as a representative ligand due to a number of recent reports detailing the synthesis of octahedral $\left[\mathrm{Co}^{\mathrm{III}}\left(\mathrm{Sal}^{\mathrm{R}}\right)(\mathrm{L})_{2}\right]^{+}$complexes with imidazole donors. ${ }^{45-47}$ The syntheses of $\left[\mathrm{Co}^{\mathrm{III}}\left(\mathrm{Sal}^{\mathrm{R}}\right)(1-\mathrm{MeIm})_{2}\right]\left[\mathrm{ClO}_{4}\right]$ (1-6) are detailed in Scheme 1, employing R groups of differing electron-donating ability. For compounds 1-5 the synthesis proceeded from isolated square planar $\mathrm{Co}^{\mathrm{II}} \mathrm{Sal}^{\mathrm{R}}$ complexes to afford the $\mathrm{Co}^{\mathrm{III}}$ products in moderate yield via reaction with 3 eq. of 1-MeIm under aerated conditions. Interestingly, the $\mathrm{Co}^{\mathrm{II}} \mathrm{Sal}^{\mathrm{CF} 3}$ precursor was difficult to isolate, likely due to the increased Lewis acidity of the $\mathrm{Co}^{\mathrm{II}}$ centre combined with the enhanced solubility of this complex. Thus, the synthesis of $\left[\mathrm{Co}^{\mathrm{III}}\left(\mathrm{Sal}^{\mathrm{CF} 3}\right)(1-\mathrm{MeIm})_{2}\right]\left[\mathrm{ClO}_{4}\right](6)$ was completed using a one-pot procedure initially reacting $\mathrm{H}_{2} \mathrm{Sal}^{\mathrm{CF} 3}$ with $\mathrm{Co}^{\mathrm{II}}$, and then subsequent addition of 1-MeIm (Scheme 1). The resulting complexes 1-6 were characterized by a number of methods including ${ }^{1} \mathrm{H}$ and ${ }^{13} \mathrm{C}$ NMR, MS, elemental analysis, X-ray crystallography for $\mathbf{1}, \mathbf{2}$, and $\mathbf{6}$.

Scheme 1. Synthesis of $\mathrm{Co}^{\mathrm{III}} \mathrm{Sal}^{\mathrm{R}}$ complexes with 1-methylimidazole (1-MeIm) as axial ligands. (Path A) 3 eq. 1-MeIm, $\mathrm{NaClO}_{4} \mathrm{MeOH} / \mathrm{CH}_{2} \mathrm{Cl}_{2}, 12 \mathrm{~h}, 298 \mathrm{~K}$. (Path B) 1 eq. $\mathrm{CoCl}_{2}, \mathrm{~N}_{2}$, and then 3 eq. 1-MeIm, $\mathrm{NaClO}_{4}, \mathrm{MeOH} / \mathrm{CH}_{2} \mathrm{Cl}_{2}, 12 \mathrm{~h}, 298 \mathrm{~K}$. See experimental section for further details.

The solid state structures of $\mathbf{1}, \mathbf{2}$, and $\mathbf{6}$ exhibit a distorted octahedral $\mathrm{N}_{4} \mathrm{O}_{2}$ coordination sphere around $\mathrm{Co}$ from the $\mathrm{Sal}^{\mathrm{R}}$ ligand in the equatorial plane and the two axial 1-MeIm ligands. The molecular structures of $\mathbf{1}$ and $\mathbf{2}$ are presented in the Supporting Information (Figures S1S2), with the molecular structure of $\mathbf{6}$ shown in Figure 2. The metrical parameters reported in 
this work (Table 2) are in agreement with published solid state structures of $\mathrm{Co}^{\mathrm{III}} \mathrm{Sal}^{\mathrm{R}}$ complexes with imidazole axial ligands. ${ }^{45-47}$

Figure 2. ORTEP plot of $\left[\mathrm{Co}^{\mathrm{III}}\left(\mathrm{Sal}^{\mathrm{CF} 3}\right)(1-\mathrm{MeIm})_{2}\right]\left[\mathrm{ClO}_{4}\right]$ (6) (50\% probability) using POV-Ray, excluding hydrogen atoms, $\mathrm{ClO}_{4}{ }^{-}$counterion, and $\mathrm{MeOH}$ solvent. See Table 2 for metrical parameters.

Table 2. Selected interatomic distances $[\AA]$ and angles $[\mathrm{deg}]$ for $\left[\mathrm{Co}^{\mathrm{III}}\left(\mathrm{Sal}^{\mathrm{R}}\right)(1-\mathrm{MeIm})_{2}\right]\left[\mathrm{ClO}_{4}\right]$ complexes $\mathbf{1}, \mathbf{2}$, and $\mathbf{6}$.

\begin{tabular}{|c|c|c|c|}
\hline$\left[\mathrm{Co}^{\prime \prime \prime}\left(\right.\right.$ salen $\left.\left.^{\mathrm{R}}\right)(1-\mathrm{Melm})_{2}\right] \mathrm{ClO}_{4}$ & $\mathrm{R}=\mathrm{H}(1)$ & $\mathrm{R}=\mathrm{OMe}(2)$ & $\mathrm{R}=\mathrm{CF}_{3}(\mathbf{6})$ \\
\hline $\mathrm{Co} 1-\mathrm{O} 1$ & $1.893(3)$ & $1.893(9)$ & $1.897(3)$ \\
\hline $\mathrm{Co} 1-\mathrm{O} 2$ & $1.888(4)$ & $1.907(9)$ & $1.908(3)$ \\
\hline Co1-N1 & $1.894(4)$ & $1.897(10)$ & $1.895(4)$ \\
\hline Co1 $1-\mathrm{N} 2$ & $1.906(5)$ & $1.893(10)$ & $1.894(4)$ \\
\hline Co1 - N3 & $1.968(4)$ & $1.943(10)$ & $1.949(4)$ \\
\hline Co1-N5 & $1.938(4)$ & $1.949(10)$ & $1.955(4)$ \\
\hline $\mathrm{O} 2-\mathrm{Co} 1-\mathrm{N} 5$ & $88.5(2)$ & $88.69(4)$ & $90.0(15)$ \\
\hline $\mathrm{N} 1-\mathrm{Co} 1-\mathrm{N} 5$ & $89.2(2)$ & $92.05(4)$ & $91.9(17)$ \\
\hline $\mathrm{N} 2-\mathrm{Co} 1-\mathrm{N} 5$ & $91.0(2)$ & $89.42(4)$ & $90.7(17)$ \\
\hline $\mathrm{O} 1-\mathrm{C} 01-\mathrm{N} 5$ & $89.7(2)$ & $89.27(4)$ & $88.6(15)$ \\
\hline $\mathrm{O} 2-\mathrm{Co} 1-\mathrm{N} 3$ & $90.1(2)$ & $87.30(4)$ & $89.6(16)$ \\
\hline $\mathrm{N} 1-\mathrm{Co} 1-\mathrm{N} 3$ & $92.2(2)$ & $91.99(4)$ & $88.6(17)$ \\
\hline $\mathrm{N} 2-\mathrm{Co} 1-\mathrm{N} 3$ & $90.1(2)$ & $91.62(4)$ & $92.4(18)$ \\
\hline $\mathrm{O} 1-\mathrm{C} 01-\mathrm{N} 3$ & $89.2(2)$ & $89.71(4)$ & $88.3(15)$ \\
\hline
\end{tabular}

The solid state data allows for investigation of the influence of the para-ring substituents on the coordination sphere bond lengths. Analysis of $\mathrm{X}$-ray distances for square planar $\mathrm{Cu}^{\mathrm{II}} \mathrm{Sal}^{\mathrm{R}}$ derivatives have shown a small but significant decrease in $\mathrm{Cu}-\mathrm{O}$ bond lengths $\left(\mathrm{CF}_{3}>\mathrm{tBu}>\right.$ $\mathrm{OMe})$ as the electron donating ability of the para-ring substituent is increased. ${ }^{48-50}$ The same trend in $\mathrm{Ni}-\mathrm{O}$ bond lengths is also observed for square planar $\mathrm{Ni}^{\mathrm{II}} \mathrm{Sal}^{\mathrm{R}}$ complexes. ${ }^{32,48-49}$ However, comparison of the Co-O distances for each of the complexes (Table 2) does not show a statistically significant change across the three complexes. In addition, no obvious trend is apparent in the Co-N distances, either equatorial or axial, in the three complexes. Thus, the 
electronic effect of the para-ring substituents do not significantly influence the coordination sphere bond lengths for octahedral $\mathrm{Co}^{\mathrm{III}} \mathrm{Sal}^{\mathrm{R}}$ complexes.

The stability of complexes 1-6 were investigated by ${ }^{1} \mathrm{H}$ NMR in $2 \%$ DMSO- $d_{6}$ in deuterated phosphate-buffered saline (PBS) solution over $24 \mathrm{~h}$ at $37{ }^{\circ} \mathrm{C}$. At $24 \mathrm{~h}$ minor signals attributable to free 1-MeIm appear for 1-4, indicating ca. 10\% ligand exchange based on integrated peak intensity (See Figures S3-S8). Thus axial ligand exchange occurs relatively slowly for 1-4 under the test conditions. Interestingly, for the $\mathrm{R}=\mathrm{NO}_{2}$ (5) and $\mathrm{R}=\mathrm{CF}_{3}$ (6) derivatives no ligand exchange is observed at $24 \mathrm{~h}$ by ${ }^{1} \mathrm{H} \mathrm{NMR}$, indicating that the complexes with electron-withdrawing para-ring substituents are more stable to axial ligand exchange. The increased stability is likely due to the enhanced Lewis acidity of the $\mathrm{Co}^{\mathrm{III}}$ centre as a result of the electron-withdrawing effect of these para-ring substituents. ${ }^{31}$

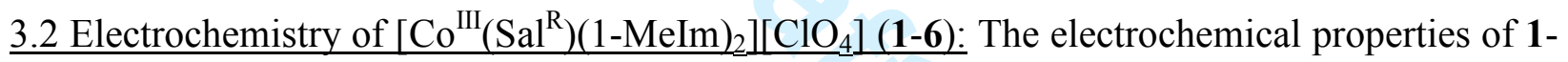
6 were assessed via cyclic voltammetry (CV) to better understand how the different parasubstituents shift the $\mathrm{Co}^{\mathrm{III}} / \mathrm{Co}^{\mathrm{II}}$ reduction potential. As shown in Figure $\mathbf{3}$ and Table $\mathbf{3}$ there is a large value of $\Delta E_{\mathrm{p}}$ (peak-to-peak difference, $\left.\Delta E_{\mathrm{p}=}\left|E_{\mathrm{a}}-E_{\mathrm{c}}\right|\right)$ and a different current response for the reduction $\left(E_{\mathrm{c}}\right)$ and oxidation $\left(E_{\mathrm{a}}\right)$ processes. These results support an irreversible electrochemical process for 1-6, and thus reduction $\left(E_{\mathrm{c}}\right)$ suggests significant geometrical re-arrangement, including axial ligand loss to afford a $\mathrm{Co}^{\mathrm{II}} \mathrm{Sal}^{\mathrm{R}}$ complex. Subsequent re-oxidation of $\mathrm{Co}^{\mathrm{II}} \mathrm{Sal}^{\mathrm{R}}\left(E_{\mathrm{a}}\right)$ is consistent with independent electrochemical analysis of the $\mathrm{Co}^{\mathrm{II}} \mathrm{Sal}^{\mathrm{R}}$ complexes (Figure S9), as the $E_{\mathrm{a}}$ values are consistent with those reported in Table 3. 
Figure 3. Cyclic voltammetry of $\left[\mathrm{Co}^{\mathrm{III}}\left(\mathrm{Sal}^{\mathrm{R}}\right)(1-\mathrm{MeIm})_{2}\right]\left[\mathrm{ClO}_{4}\right]$ (1-6). Conditions: $1 \mathrm{mM}$ complex, $0.1 \mathrm{M}^{\mathrm{n}} \mathrm{Bu}_{4} \mathrm{NClO}_{4}$, scan rate $100 \mathrm{mV} \mathrm{s}^{-1}$, DMF, $298 \mathrm{~K}$. Arrow signifies scan direction. Inset: Hammett plot of the $E_{1 / 2}$ value of $\left[\mathrm{Co}^{\mathrm{III}}\left(\mathrm{Sal}^{\mathrm{R}}\right)(1-\mathrm{MeIm})_{2}\right]\left[\mathrm{ClO}_{4}\right](\mathbf{1 - 6})$ versus $\sigma_{\text {para }}$ of the para-ring substituents.

Table 3. Electrochemical data $(\mathrm{mV})$ for $\left[\mathrm{Co}^{\mathrm{III}}\left(\mathrm{Sal}^{\mathrm{R}}\right)(1-\mathrm{MeIm})_{2}\right]\left[\mathrm{ClO}_{4}\right](\mathbf{1 - 6})$ versus NHE.

\begin{tabular}{cllll}
\hline$\left[\mathrm{Co}\left(\mathrm{salen}^{\mathrm{R}}\right)(1-\mathrm{MeIm})_{2}\right] \mathrm{ClO}_{4}$ & $E_{\mathrm{c}}$ & $E_{\mathrm{a}}$ & $E_{1 / 2}$ & $\Delta E_{\mathrm{p}}{ }^{\mathrm{a}}$ \\
$\mathrm{OMe} \mathrm{(2)}$ & -540 & 260 & -140 & 800 \\
${ }^{t} \mathrm{Bu}(\mathbf{3})$ & -570 & 320 & -125 & 890 \\
$\mathrm{H}(\mathbf{1})$ & -490 & 290 & -100 & 780 \\
$\mathrm{Br}(\mathbf{4})$ & -400 & 300 & -50 & 700 \\
$\mathrm{CF}_{3}(\mathbf{6})$ & -420 & 470 & 25 & 790 \\
$\mathrm{NO}_{2}(\mathbf{5})$ & -230 & 390 & 80 & 620 \\
\hline
\end{tabular}

${ }^{\text {a }}$ Peak-to-peak differences $\left(\Delta E_{\mathrm{p}=}\left|E_{\mathrm{a}}-E_{\mathrm{c}}\right|\right)$. Peak-to-peak difference for the $\mathrm{Fc}^{+} / \mathrm{Fc}$ couple at $298 \mathrm{~K}$ is $130 \mathrm{mV}$

The trend in reduction potential $\left(E_{1 / 2}\right)$ for 1-6 follows the relative electronic properties of the phenolate para-substituents since the donating para-ring substituents afford a lower $\mathrm{Co}^{\mathrm{III}} /$ $\mathrm{Co}^{\mathrm{II}}$ reduction potential due to the increased electron density at the Co centre. Overall, using the different para-ring substituents allows for tuning of the $E_{1 / 2}$ value from $-140 \mathrm{mV}$ to $80 \mathrm{mV}$ (220 $\mathrm{mV}$ difference), and $E_{\mathrm{c}}$ value from $-570 \mathrm{mV}$ to $-230 \mathrm{mV}$ (340 $\mathrm{mV}$ difference). Plotting the $E_{1 / 2}$ values against the Hammett substituent constants $\left(\sigma_{\text {para }}\right)^{51-52}$ as shown in Figure 3 (Inset) demonstrates that the reduction potential is predominantly affected by the donating ability of the para-ring substituents. These promising results show that it is possible to significantly alter the $\mathrm{Co}^{\mathrm{III}} / \mathrm{Co}^{\mathrm{II}}$ reduction potential and, in addition, reduction leads to significant geometrical rearrangement and axial ligand loss.

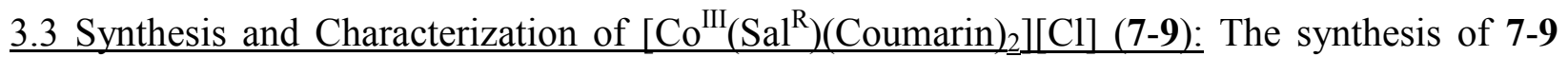
followed closely to that of $\mathbf{6}$, using a one-pot procedure to first synthesize the $\mathrm{Co}^{\mathrm{II}} \mathrm{Sal}^{\mathrm{R}}$ complex in situ, and then oxidation in the presence of 2 eq. of the Coumarin ligand (Scheme 2). The 
Coumarin ligand was chosen as a reporter due to its known fluorescence properties, and was coupled to the imidazole moiety for coordination to Co. Encouragingly, upon binding to the Co ${ }^{\text {III }}$ centre the Coumarin fluorescence is quenched (see below). Use of a quenched fluorophore allows for the rational optimization of ligand release under physiological conditions, and monitoring of release under hypoxic conditions, and in cells. We initially focused on complexes incorporating the $\mathrm{ClO}_{4}{ }^{-}$counterion, however, the resulting products were difficult to purify and exhibited limited solubility in water. Employing $\mathrm{CoCl}_{2}$ as the starting material, and without addition of $\mathrm{NaClO}_{4}$, we were able to isolate 7-9 in moderate yields (Scheme 2). These three complexes span a large range of electron-donating abilities $\left(\mathrm{OMe}, \mathrm{H}\right.$, and $\left.\mathrm{CF}_{3}\right)$, thus affording a representative series of complexes for further analysis.

Scheme 2. Synthesis of $\mathrm{Co}^{\mathrm{III}} \mathrm{Sal}^{\mathrm{R}}$ complexes with Coumarin-imidazole conjugates (Coumarin) as axial ligands. Conditions: 1 eq. $\mathrm{CoCl}_{2}, \mathrm{MeOH} / \mathrm{CH}_{2} \mathrm{Cl}_{2}$, under $\mathrm{N}_{2}$, then 3 eq. Coumarin, 12 h, 298 $\mathrm{K}$. See experimental section for further details.

The solid state structure of $\mathbf{9}$ is shown in Figure 4, and exhibits a distorted octahedral $\mathrm{N}_{4} \mathrm{O}_{2}$ coordination sphere at $\mathrm{Co}$ with the $\mathrm{Sal}^{\mathrm{CF} 3}$ ligand in the equatorial plane and the two axial Coumarin ligands. The pattern of first-coordination sphere bond lengths for 9 is similar to that reported for complexes $\mathbf{1 , 2}$, and $\mathbf{6}$, reflecting the common ligating groups.

Figure 4. ORTEP plot of $\left[\mathrm{Co}^{\mathrm{III}}\left(\mathrm{Sal}^{\mathrm{CF} 3}\right)(\mathrm{Coumarin})_{2}\right][\mathrm{Cl}]$ (9) (50\% probability) using POV-Ray, excluding hydrogen atoms and disordered $\mathrm{Cl}$ counterion. Note significant disorder is present for one coumarin and the $\mathrm{CF}_{3}$ substituents. Selected interatomic distances $[\AA]$ and angles [deg]: Co1-O1 1.901(2), Co1-O2 1.887(3), Co1-N1 1.891(2), Co1-N2 1.895(4), Co1-N3 1.946(4), Co1N5 2.018(3); angles: O2-Co1-N5 88.4(12), N1-Co1-N5 92.7(4), N2-Co1-N5 94.3(3), O1-Co1N5 86.2(12), O2-Co1-N3 89.2(17), N1-Co1-N3 89.8(18), N2-Co1-N3 91.7(18), O1-Co1-N3 87.8(16), O1-Co1-N2 179.5(18),O2-Co1-N1 179.0(18). 
The stability of the $\left[\mathrm{Co}^{\mathrm{III}}\left(\mathrm{Sal}^{\mathrm{R}}\right)(\mathrm{Coumarin})_{2}\right][\mathrm{Cl}](\mathbf{7 - 9})$ complexes was investigated by ${ }^{1} \mathrm{H}$ NMR in $50 \%$ DMSO- $d_{6}$ in $\mathrm{D}_{2} \mathrm{O}$ over $72 \mathrm{~h}$ at $37{ }^{\circ} \mathrm{C}$. The increased amount of DMSO used here in comparison to the ${ }^{1} \mathrm{H}$ NMR stability studies for 1-6 was necessary due to the lower aqueous solubility of the Coumarin derivatives. In each case, compounds 7-9 displayed no obvious sign of decomposition over the $72 \mathrm{~h}$ period (Figures S11-S13).

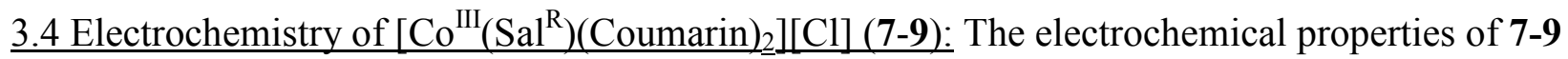
were assessed via CV to determine reduction potentials and possibility of axial ligand loss upon reduction. As shown in Figure 5 and Table 4, complexes 7-9 exhibit a large value of $\Delta E_{\mathrm{p}}$ and a different current response for the reduction $\left(E_{\mathrm{c}}\right)$ and oxidation $\left(E_{\mathrm{a}}\right)$ processes. These results suggest a similar electrochemical response for 7-9 in comparison to 1-6 consistent with significant geometrical re-arrangement, including axial ligand loss upon reduction. The $E_{1 / 2}$ values follow a trend consistent with the relative electron-donating ability of the para-ring substituents $\left(\mathrm{OMe}>\mathrm{H}>\mathrm{CF}_{3}\right)$. Interestingly, the $E_{1 / 2}$ values are ca. $80 \mathrm{mV}$ more positive in comparison to the $\left[\mathrm{Co}^{\mathrm{III}}\left(\mathrm{Sal}^{\mathrm{R}}\right)(1-\mathrm{MeIm})_{2}\right]\left[\mathrm{ClO}_{4}\right]$ derivatives with the same $\mathrm{Sal}^{\mathrm{R}}$ ligands. The shift indicates that the $E_{1 / 2}$ value depends slightly on the axial ligand and the counterion in this experiment.

Figure 5. Cyclic voltammetry of $\left[\mathrm{Co}^{\mathrm{III}}\left(\mathrm{Sal}^{\mathrm{R}}\right)(\text { Coumarin })_{2}\right][\mathrm{Cl}]$ (7-9). Conditions: $1 \mathrm{mM}$ complex, $0.1 \mathrm{M}^{\mathrm{n}} \mathrm{Bu}_{4} \mathrm{NClO}_{4}$, scan rate $100 \mathrm{mV} \mathrm{s}^{-1}$, DMF, $298 \mathrm{~K}$. Arrow signifies scan direction. 
Table 4. Electrochemical data $(\mathrm{mV})$ for $\left[\mathrm{Co}^{\mathrm{III}}\left(\mathrm{Sal}^{\mathrm{R}}\right)(\mathrm{Coumarin})_{2}\right][\mathrm{Cl}](\mathbf{7 - 9})$ versus NHE.

\begin{tabular}{cllll}
\hline [Co'"(salen ${ }^{\mathrm{R}}$ )(Coumarin) $\left.)_{2}\right] \mathrm{Cl}$ & $E_{c}$ & $E_{a}$ & $E_{1 / 2}$ & $\Delta E_{\mathrm{p}}{ }^{\mathrm{a}}$ \\
$\mathrm{R}=\mathrm{OMe}(\mathbf{8})$ & -390 & 270 & -60 & 660 \\
$\mathrm{R}=\mathrm{H}(\mathbf{7})$ & -310 & 270 & -20 & 580 \\
$\mathrm{R}=\mathrm{CF}_{3}(\mathbf{9})$ & -280 & 370 & 45 & 650 \\
\hline
\end{tabular}

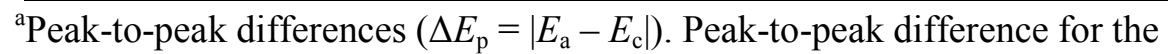
$\mathrm{Fc}^{+} / \mathrm{Fc}$ couple at $298 \mathrm{~K}$ is $120 \mathrm{mV}$.

3.5 Emission studies of $\left[\mathrm{Co}^{\mathrm{III}}\left(\mathrm{Sal}^{\mathrm{R}}\right)(\mathrm{Coumarin})_{2}\right][\mathrm{Cl}](7-9):$ We next assessed the use of fluorescence to monitor the rate of ligand loss from compounds 7-9 upon reduction in solution. The photophysical properties of the Coumarin ligand were first investigated, with an optimal excitation wavelength $\left(\lambda_{\mathrm{ex}}\right)$ of $327 \mathrm{~nm}$ and emission wavelength $\left(\lambda_{\mathrm{em}}\right)$ of $400 \mathrm{~nm}$ (Figure S14). Upon binding to the $\mathrm{Co}^{\mathrm{III}}$ centre the Coumarin ligand emission is quenched (Figure 6), likely due to excited state energy-transfer. ${ }^{53-55}$ A fluorescence signal is observed however for the $\mathrm{R}=\mathrm{H}$ (7, ca. $3 \%$ of concentration-matched Coumarin signal) and $\mathrm{R}=\mathrm{OMe}(\mathbf{8}, \mathrm{ca} .14 \%$ of concentration-matched Coumarin signal) analogues upon dissolution, likely due to partial axial ligand exchange in the solvent medium. Interestingly, essentially no fluorescence signal is observed for the $\mathrm{R}=\mathrm{CF}_{3}$ derivative $(\mathbf{9}$, ca. $0.4 \%$ of concentration-matched Coumarin signal).

Figure 6. Emission spectra of Coumarin (dash-dot), 7 (black line), 8 (grey dashed), and 9 (dotted). Conditions: $2 \%$ DMSO in PBS $(0.1 \mathrm{M}), 298 \mathrm{~K}, \lambda_{\mathrm{ex}}=327 \mathrm{~nm}$.

The ligand exchange processes of 7-9 were studied in the presence of excess dithionite $\left(\mathrm{Na}_{2} \mathrm{~S}_{2} \mathrm{O}_{4}\right)$. The reduction potential for dithionite $\left(E_{1 / 2}=-660 \mathrm{mV} \text { vs. NHE }\right)^{56}$ is considerably lower than 7-9 (Table 4), and thus we expected this reagent to reduce the $\mathrm{Co}^{\mathrm{III}}$ complexes. As this reagent readily reacts with $\mathrm{O}_{2}$ in solution, solutions were degassed (purging with $\mathrm{N}_{2}$ ) before a 10 -fold excess of $\mathrm{Na}_{2} \mathrm{~S}_{2} \mathrm{O}_{4}$ was added to ensure reduction of 7-9. ${ }^{56-58}$ We then compared the 
change in fluorescence of 7-9 in solution in the presence of excess $\mathrm{Na}_{2} \mathrm{~S}_{2} \mathrm{O}_{4}$, excess 1-MeIm, and finally without any additives.

The change in fluorescence of $7(3 \mu \mathrm{M})$ over a 12 h period was measured: (i) on its own in solution, (ii) with $30 \mu \mathrm{M}$ dithionite, and (iii) with $30 \mu \mathrm{M}$ 1-MeIm (Figure 7). Coumarin fluorescence was used as the control, and was shown to be unaffected by either $\mathrm{Na}_{2} \mathrm{~S}_{2} \mathrm{O}_{4}$, or 1MeIm. Over a period of $12 \mathrm{~h}$ compound 7 exhibited a ca. $60 \%$ increase (relative to Coumarin control) in fluorescence in the presence of excess $\mathrm{Na}_{2} \mathrm{~S}_{2} \mathrm{O}_{4}$. In the presence of excess 1-MeIm, a ca. $44 \%$ increase in fluorescence is observed, indicating a significant amount of axial ligand exchange. However, a higher fluorescence response is observed under reducing conditions. In the absence of both reducing agent and 1-MeIm, the complex also released Coumarin (ca. $27 \%$ of Coumarin control), suggesting ligand exchange in the buffer solution.

Figure 7. Fluorescence analysis of solutions of $7(3 \mu \mathrm{M})$ in the presence of $30 \mu \mathrm{M} \mathrm{Na} \mathrm{Na}_{2} \mathrm{O}_{4}$ (black squares), $30 \mu \mathrm{M}$ 1-MeIm (black triangles), and without additives (black circles) over a period of $12 \mathrm{~h}$. The Coumarin standard $(6 \mu \mathrm{M})$ is shown as grey circles. Conditions: 2\% DMSO in PBS (0.1 M), $298 \mathrm{~K}, \lambda_{\text {ex }}=327 \mathrm{~nm}, \lambda_{\mathrm{em}}=400 \mathrm{~nm}$.

The change in fluorescence of $\mathbf{8}$ over a $12 \mathrm{~h}$ period under the same conditions is shown in Figure 8. The overall response to $\mathrm{Na}_{2} \mathrm{~S}_{2} \mathrm{O}_{4}$ (ca. $67 \%$ of Coumarin control), excess 1-MeIm (ca. $44 \%$ of Coumarin control), and buffer (ca. $26 \%$ of control) are similar to that described for compound 7. However, the fluorescence increases occur in the first $2 \mathrm{~h}$, and from $2-12 \mathrm{~h}$ the fluorescence readings remain relatively constant. 
Figure 8. Fluorescence analysis of solutions of $8(3 \mu \mathrm{M})$ in the presence of $30 \mu \mathrm{M} \mathrm{Na} \mathrm{Na}_{2} \mathrm{O}_{4}$ (black squares), $30 \mu \mathrm{M}$ 1-MeIm (black triangles), and without additives (black circles) over a period of $12 \mathrm{~h}$. The Coumarin standard $(6 \mu \mathrm{M})$ is shown as grey circles. Conditions: 2\% DMSO in PBS (0.1 M), $298 \mathrm{~K}, \lambda_{\mathrm{ex}}=327 \mathrm{~nm}, \lambda_{\mathrm{em}}=400 \mathrm{~nm}$.

As shown in Figure 9, complex 9 exhibited a minimal increase in fluorescence under the test conditions over $12 \mathrm{~h}$. An expanded view of the fluorescence plot shows that while small, the fluorescence intensity increase is greater in the presence of $\mathrm{Na}_{2} \mathrm{~S}_{2} \mathrm{O}_{4}$ in comparison to 1-MeIm and buffer alone. Indeed, the fluorescence intensity of 9 in buffer is the same as that in the presence of excess 1-MeIm, indicating minimal ligand exchange for 9.

Figure 9. Fluorescence analysis of solutions of $9(3 \mu \mathrm{M})$ in the presence of $30 \mu \mathrm{M} \mathrm{Na} \mathrm{Na}_{2} \mathrm{O}_{4}$ (black squares), $30 \mu \mathrm{M}$ 1-MeIm (black triangles), and without additives (black circles) over a period of $12 \mathrm{~h}$. The Coumarin standard $(6 \mu \mathrm{M})$ is shown as grey circles. Conditions: 2\% DMSO in PBS $(0.1 \mathrm{M}), 298 \mathrm{~K}, \lambda_{\mathrm{ex}}=327 \mathrm{~nm}, \lambda_{\mathrm{em}}=400 \mathrm{~nm}$. Inset: Expanded view of the fluorescence increase in the presence of $30 \mu \mathrm{M} \mathrm{Na}_{2} \mathrm{~S}_{2} \mathrm{O}_{4}$ (black squares), $30 \mu \mathrm{M}$ 1-MeIm (black triangles).

The rate of axial ligand exchange (without added reductant) for 7-9 can be rationalized by the effect of the para-ring substituents on the Lewis acidity at the metal centre. The electronwithdrawing $\mathrm{R}=\mathrm{CF}_{3}$ substituent is expected to increase the Lewis acidity at the metal centre to the greatest extent $\left(\mathrm{CF}_{3}>\mathrm{H}>\mathrm{OMe}\right)$, resulting in increased bond strength between the $\mathrm{Co}^{\mathrm{III}}$ centre and the axial ligands. This is reflected in the rate of exchange of the Co ${ }^{\mathrm{III}}$ complexes in the presence of 1-MeIm $\left(\mathrm{OMe}>\mathrm{H}>\mathrm{CF}_{3}\right)$ as shown in Figures 7-9. These results are consistent with a literature report ${ }^{59}$ that demonstrated increased resistance to ligand exchange (via reduced enzyme inhibition) for Co N,N'ethylenebis(acetylacetoneiminate) complexes when electronwithdrawing groups are installed on the ligand. Interestingly, the observed rates $(\mathrm{OMe}>\mathrm{H}>$ $\mathrm{CF}_{3}$ ) of reduction in the presence of excess $\mathrm{Na}_{2} \mathrm{~S}_{2} \mathrm{O}_{4}$ are opposite to that expected based on the reduction potentials (Table 4), however the potential window is relatively narrow for the three 
derivatives (-0.06 to $0.45 \mathrm{~V}$ vs. NHE). The reduction processes studied in this work are relatively slow (timescale of h), which agrees with reports of similar Co complexes in the literature. ${ }^{10,25,60-}$ 62 Due to the slow reduction process, and reaction conditions (in air), in which $\mathrm{Na}_{2} \mathrm{~S}_{2} \mathrm{O}_{4}$ decomposes, the observed fluorescence increase is likely a combination of both reduction and axial ligand exchange.

\section{Summary:}

In this work we have studied the electrochemical properties and axial ligand exchange of a series of octahedral $\mathrm{Co}^{\mathrm{III}}$ salen $\left(\mathrm{Sal}^{\mathrm{R}}\right)$ complexes. We first focused on tuning the $\mathrm{Co}^{\mathrm{III}} / \mathrm{Co}^{\mathrm{II}}$ reduction potential of 1-6 using a series of $\mathrm{Sal}^{\mathrm{R}}$ ligands with para-ring substituents of different electrondonating abilities $\left(\mathrm{H}, \mathrm{OMe}, \mathrm{tBu}, \mathrm{Br}, \mathrm{NO}_{2}, \mathrm{CF}_{3}\right)$. Using peripheral ligand electronics we were able to tune the $\mathrm{Co}^{\mathrm{III}} / \mathrm{Co}^{\mathrm{II}}$ reduction potential in the range of -140 to $80 \mathrm{mV}$ vs. NHE. While at the edge of the generally-accepted potential window in cells $\left(-420\right.$ to $-150 \mathrm{mV}$ vs. NHE) ${ }^{27}$ our results suggest that it will be possible to tune the $\mathrm{Co}^{\mathrm{III}} / \mathrm{Co}^{\mathrm{II}}$ reduction potential further into a physiologically-relevant range by employing ring substituents of enhanced electron-donating ability (such as $\mathrm{NMe}_{2}$ ). ${ }^{29-32}$ In addition, using 1-MeIm axial ligands, we showed that reduction to $\mathrm{Co}^{\mathrm{II}}$ results in significant geometrical re-organization and axial ligand exchange. By installing fluorescent coumarin-imidazole conjugate (Coumarin) axial ligands, we explored the electrochemical properties and axial ligand exchange in a smaller series of octahedral $\mathrm{Co}^{\mathrm{III}}$ complexes 7-9 $\left(\mathrm{H}, \mathrm{OMe}, \mathrm{CF}_{3}\right)$. Upon binding to $\mathrm{Co}^{\mathrm{III}}$, the Coumarin fluorescence was quenched, allowing for a sensitive read-out of axial ligand exchange upon release. Our results show that both $7(\mathrm{R}=\mathrm{H})$, and $8(\mathrm{R}=\mathrm{OMe})$ exhibit substantial ligand exchange in the presence of either excess reductant or competing ligands, with the latter compound reacting much more quickly. Interestingly, compound 9 did not exhibit a substantial fluorescence change under any 
conditions, even though the $\mathrm{Co}^{\mathrm{III}} / \mathrm{Co}^{\mathrm{II}}$ reduction potential suggests the most significant driving force for reduction. Overall, the current results suggest that the dominant factor influencing axial ligand exchange is the Lewis acidity at the Co centre, and not the $\mathrm{Co}^{\mathrm{III}} / \mathrm{Co}^{\mathrm{II}}$ reduction potential. Future work will focus on decoupling these competing processes for this series of complexes.

\section{Acknowledgements:}

This work is supported by NSERC Discovery Grants to T. S. and C. J. W, and a Michael Smith Foundation for Health Research Career Investigator Award to T. S.. C. Z. acknowledges a Mitacs Globalink Fellowship, and R M. C. thanks NSERC for a postgraduate fellowship. Dr. Angela Stefanachi (Dipartimento di Farmacia, Scienze del Farmaco, Universita of Bari Aldo Moro, Italy) is thanked for information on the fluorescence of the coumarin-imidazole adduct. The Advanced Light Source (ALS) is supported by the U.S. Department of Energy, Office of Energy Sciences Materials Sciences Division, under Contract DE-AC02-05CH11231.

\section{References:}

(1) Hambley, T. W. Dalton Trans. 2007, 4929-4937.

(2) Hambley, T. W. Science 2007, 318, 1392-1393.

(3) van Rijt, S. H.; Sadler, P. J. Drug Disc. Today 2009, 14, 1089-1097.

(4) Mjos, K. D.; Orvig, C. Chem. Rev. 2014, 114, 4540-4563.

(5) Storr, T. Ligand Design in Medicinal Inorganic Chemistry; Wiley, 2014.

(6) Chiang, L.; Jones, M. R.; Ferreira, C. L.; Storr, T. Curr. Top. Med. Chem. 2012, 12, 122144.

(7) Lippert, B. Cisplatin: Chemistry and Biochemistry of a Leading Anticancer Drug; Wiley, 1999.

(8) Hall, M. D.; Failes, T. W.; Yamamoto, N.; Hambley, T. W. Dalton Trans. 2007, 3983-3990. 
(9) Pizarro, A. M.; Habtemariam, A.; Sadler, P. J. In Medicinal Organometallic Chemistry; Jaouen, G., MetzlerNolte, N., Eds. 2010; Vol. 32, p 21-56.

(10) Yamamoto, N.; Renfrew, A. K.; Kim, B. J.; Bryce, N. S.; Hambley, T. W. J. Med. Chem. 2012, 55, 11013-11021.

(11) Graf, N.; Lippard, S. J. Adv. Drug Deliv. Rev. 2012, 64, 993-1004.

(12) Mari, C.; Pierroz, V.; Ferrari, S.; Gasser, G. Chem. Sci. 2015, 6, 2660-2686.

(13) Renfrew, A. K. Metallomics 2014, 6, 1324-1335.

(14) O'Donnell, J. L.; Shannon, A. M.; Bouchier-Hayes, D. In Anticancer Therapeutics; John Wiley \& Sons, Ltd: 2008, p 245-261.

(15) Hockel, M.; Vaupel, P. J. Natl. Cancer Inst. 2001, 93, 266-276.

(16) Teicher, B. A. Cancer Metastasis Rev. 1994, 13, 139-168.

(17) Bonnitcha, P. D.; Kim, B. J.; Hocking, R. K.; Clegg, J. K.; Turner, P.; Neville, S. M.; Hambley, T. W. Dalton Trans. 2012, 41, 11293-11304.

(18) Hartinger, C. G.; Zorbas-Seifried, S.; Jakupec, M. A.; Kynast, B.; Zorbas, H.; Keppler, B. K. J. Inorg. Biochem. 2006, 100, 891-904.

(19) Reisner, E.; Arion, V. B.; Keppler, B. K.; Pombeiro, A. J. L. Inorg. Chim. Acta 2008, 361, 1569-1583.

(20) Webb, M. I.; Walsby, C. J. Metallomics 2013, 5, 1624-1633.

(21) Blazevic, A.; Hummer, A. A.; Heffeter, P.; Berger, W.; Filipits, M.; Cibin, G.; Keppler, B. K.; Rompel, A. Sci. Rep. 2017, 7, 40966.

(22) Ware, D. C.; Palmer, H. R.; Brothers, P. J.; Rickard, C. E. F.; Wilson, W. R.; Denny, W. A. J. Inorg. Biochem. 1997, 68, 215-224.

(23) Lippard, S. J.; Berg, J. M. Principles of Bioinorganic Chemistry; University Science Books: Mill Valley, CA, 1994.

(24) Failes, T. W.; Cullinane, C.; Diakos, C. I.; Yamamoto, N.; Lyons, J. G.; Hambley, T. W. Chem. Eur. J. 2007, 13, 2974-2982.

(25) Renfrew, A. K.; Bryce, N. S.; Hambley, T. W. Chem. Sci. 2013, 4, 3731-3739.

(26) Denny, A. D. Future Oncol. 2010, 6, 419-428.

(27) Go, Y. M.; Jones, D. P. Free Radical Biol. Med. 2011, 50, 495-509.

(28) Kizaka-Kondoh, S.; Inoue, M.; Harada, H.; Hiraoka, M. Cancer Sci. 2003, 94, 1021-1028. 
(29) Herasymchuk, K.; Chiang, L.; Hayes, C. E.; Brown, M. L.; Ovens, J. S.; Patrick, B. O.; Leznoff, D. B.; Storr, T. Dalton Trans. 2016, 45, 12576-12586.

(30) Clarke, R. M.; Storr, T. J. Am. Chem. Soc. 2016, 138, 15299-15302.

(31) Chiang, L.; Allan, L. E.; Alcantara, J.; Wang, M. C.; Storr, T.; Shaver, M. P. Dalton Trans. 2014, 43, 4295-4304.

(32) Chiang, L.; Kochem, A.; Jarjayes, O.; Dunn, T. J.; Vezin, H.; Sakaguchi, M.; Ogura, T.; Orio, M.; Shimazaki, Y.; Thomas, F.; Storr, T. Chem. Eur. J. 2012, 18, 14117-14127.

(33) Gust, R.; Ott, I.; Posselt, D.; Sommer, K. J. Med. Chem. 2004, 47, 5837-5846.

(34) Ali, A.; Kamra, M.; Bhan, A.; Mandal, S. S.; Bhattacharya, S. Dalton Trans. 2016, 45, 9345-9353.

(35) Coletti, A.; Galloni, P.; Sartorel, A.; Conte, V.; Floris, B. Catal. Today 2012, 192, 44-55.

(36) Mathavan, A.; Ramdass, A.; Rajagopal, S. Transition Met. Chem. 2015, 40, 355-362.

(37) Leonetti, F.; Favia, A.; Rao, A.; Aliano, R.; Paluszcak, A.; Hartmann, R. W.; Carotti, A. J. Med. Chem. 2004, 47, 6792-6803.

(38) Altomare, A.; Cascarano, G.; Giacovazzo, C.; Guagliardi, A. J. Appl. Crystallogr. 1993, 26, 343-350.

(39) Sheldrick, G. M. Acta Cryst. C 2015, 71, 3-8.

(40) Sheldrick, G. M.; Bruker AXS Inc.: Madison, WI..

(41) Hubschle, C. B.; Sheldrick, G. M.; Dittrich, B. J. J. Appl. Crystallogr. 2011, 44, 1281.

(42) Farrugia, L. J. J. Appl. Crystallogr. 2012, 45, 849-854.

(43) Persistence of Vision Pty. Ltd.: Victoria, Australia, 2004; Vol. 3.6.2..

(44) Fenn, T. D.; Ringe, D.; Petsko, G. A. J. Appl. Crystallogr. 2003, 36, 944.

(45) Kochem, A.; Kanso, H.; Baptiste, B.; Arora, H.; Philouze, C.; Jarjayes, O.; Vezin, H.; Luneau, D.; Orio, M.; Thomas, F. Inorg. Chem. 2012, 51, 10557-10571.

(46) Heffern, M. C.; Reichova, V.; Coomes, J. L.; Harney, A. S.; Bajema, E. A.; Meade, T. J. Inorg. Chem. 2015, 54, 9066-9074.

(47) Manus, L. M.; Holbrook, R. J.; Atesin, T. A.; Heffern, M. C.; Harney, A. S.; Eckermann, A. L.; Meade, T. J. Inorg. Chem. 2013, 52, 1069-1076.

(48) Orio, M.; Jarjayes, O.; Kanso, H.; Philouze, C.; Neese, F.; Thomas, F. Angew. Chem. Int. Ed. 2010, 49, 4989-4992. 
(49) Chiang, L.; Herasymchuk, K.; Thomas, F.; Storr, T. Inorg. Chem. 2015, 54, 5970-5980.

(50) Bunce, S.; Cross, R. J.; Farrugia, L. J.; Kunchandy, S.; Meason, L. L.; Muir, K. W.; O'Donnell, M.; Peacock, R. D.; Stirling, D.; Teat, S. J. Polyhedron 1998, 17, 4179-4187.

(51) Hansch, C.; Leo, A.; Taft, R. W. Chem. Rev. 1991, 91, 165-195.

(52) Carroll, F. A. Perspectives on Structure and Mechanism in Organic Chemistry; 2 ed.; John Wiley \& Sons, Inc., 2010.

(53) Salthammer, T.; Dreeskamp, H.; Birch, D. J. S.; Imhof, R. E. J. Photochem. Photobiol. A: Chem. 1990, 55, 53-62.

(54) Kemlo, J. A.; Shepherd, T. M. Chem. Phys. Lett. 1977, 47, 158-162.

(55) Varnes, A. W.; Wehry, E. L.; Dodson, R. B. J. Am. Chem. Soc. 1972, 94, 946-\&.

(56) Mayhew, S. G. Eur. J. Biochem. 1978, 85, 535-547.

(57) Barman, B. G.; Tollin, G. Biochemistry 1972, 11, 4755-4759.

(58) Scaife, C. W. J.; Wilkins, R. G. Inorg. Chem. 1980, 19, 3244-3247.

(59) Takeuchi, T.; Böttcher, A.; Quezada, C. M.; Meade, T. J.; Gray, H. B. Biorg. Med. Chem. 1999, 7, 815-819.

(60) Yamamoto, N.; Danos, S.; Bonnitcha, P. D.; Failes, T. W.; New, E. J.; Hambley, T. W. J. Biol. Inorg. Chem. 2008, 13, 861-871.

(61) Kim, B. J.; Hambley, T. W.; Bryce, N. S. Chem. Sci. 2011, 2, 2135-2142.

(62) Garcia, C. V.; Parrilha, G. L.; Rodrigues, B. L.; Barbeira, P. J. S.; Clarke, R. M.; Storr, T.; Beraldo, H. Polyhedron 2017, 124, 86-95. 


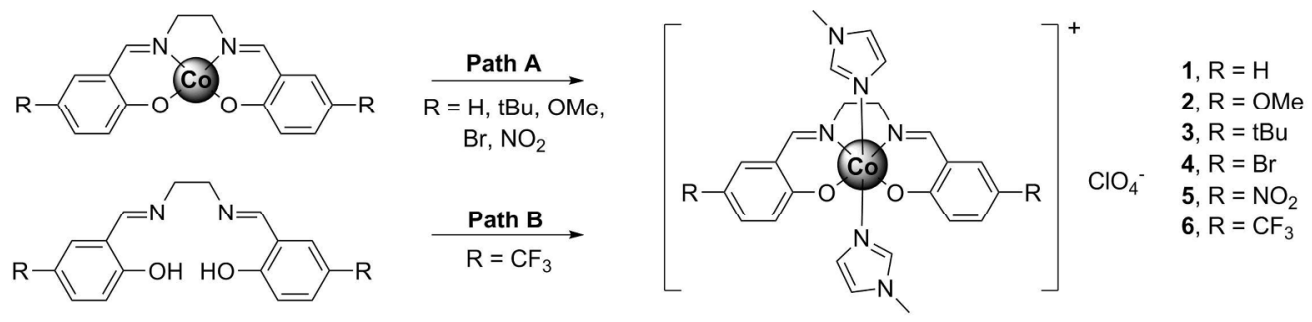

$602 \times 145 \mathrm{~mm}(96 \times 96 \mathrm{DPI})$ 


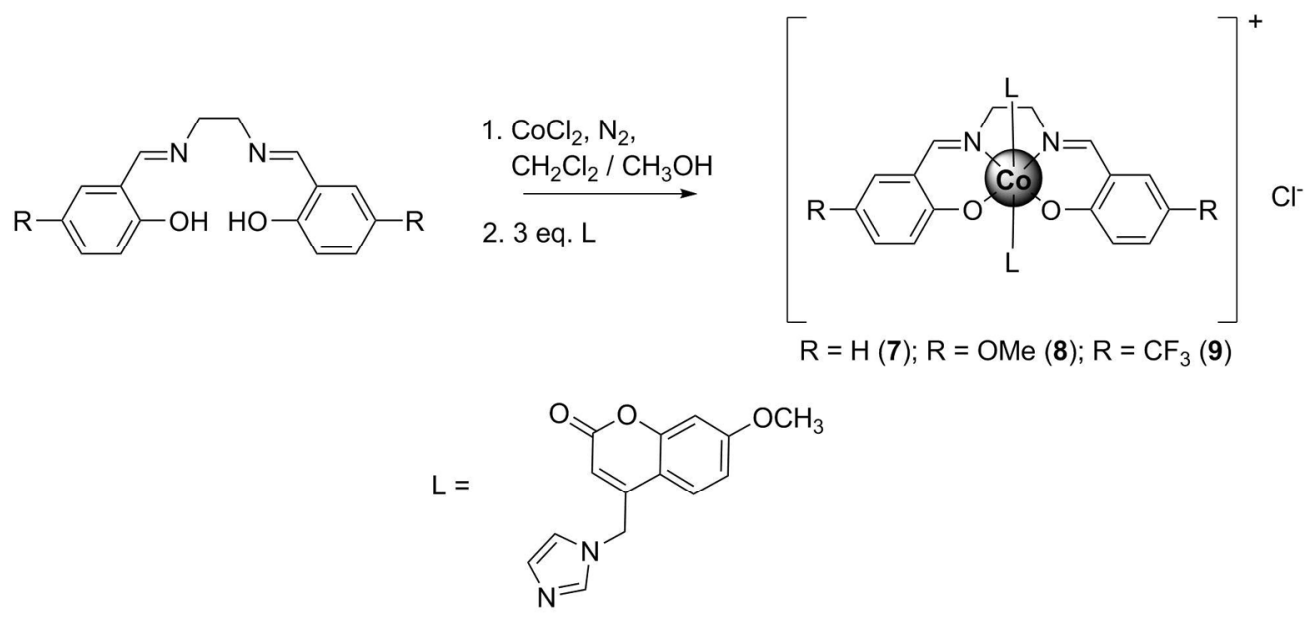

$527 \times 248 m m(96 \times 96$ DPI $)$ 
A

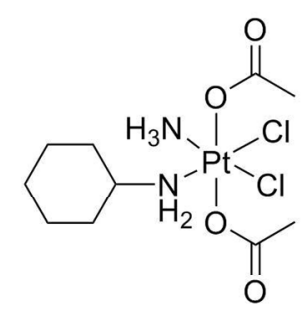

Satraplatin
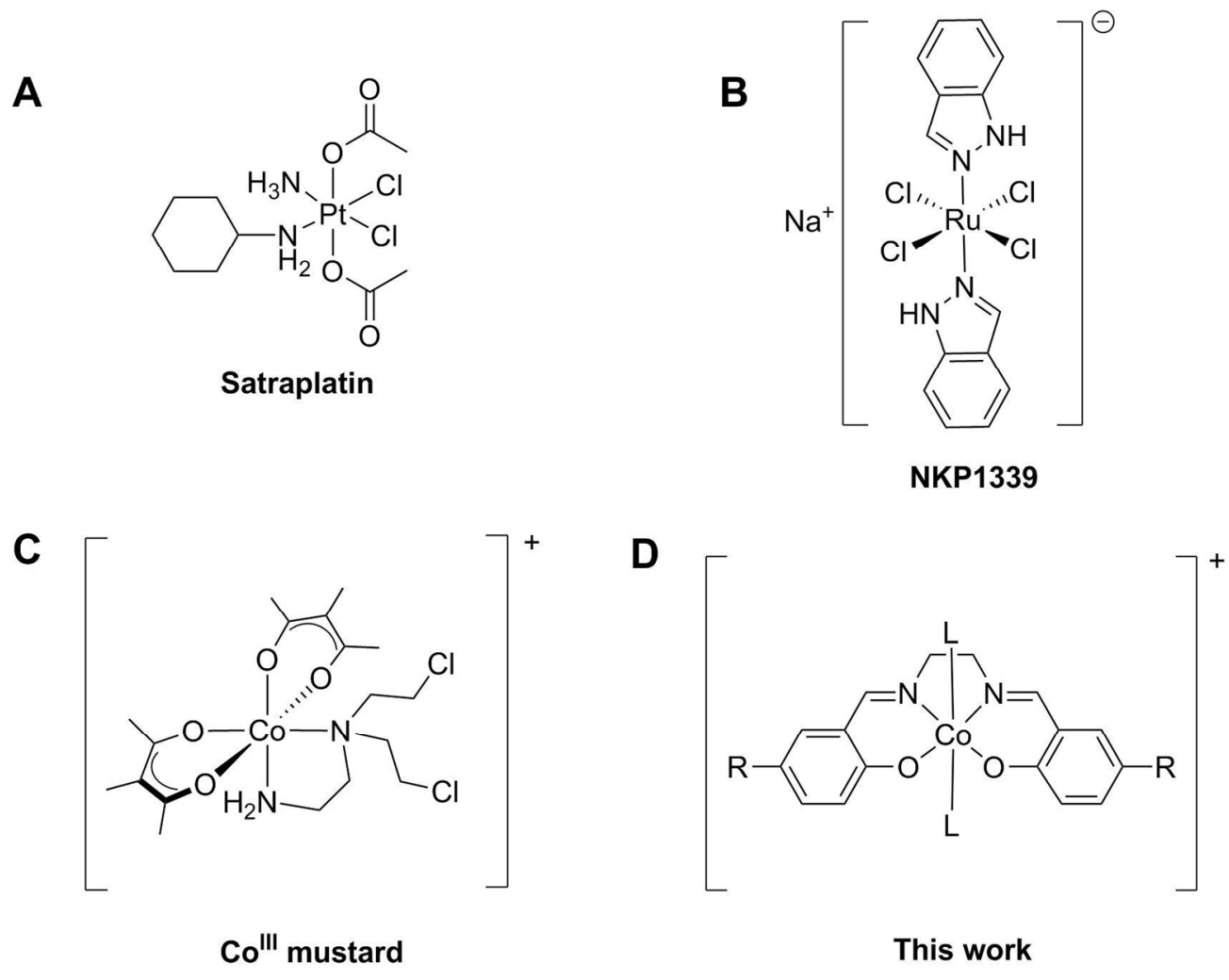

This work

$454 \times 359 \mathrm{~mm}(96 \times 96 \mathrm{DPI})$ 


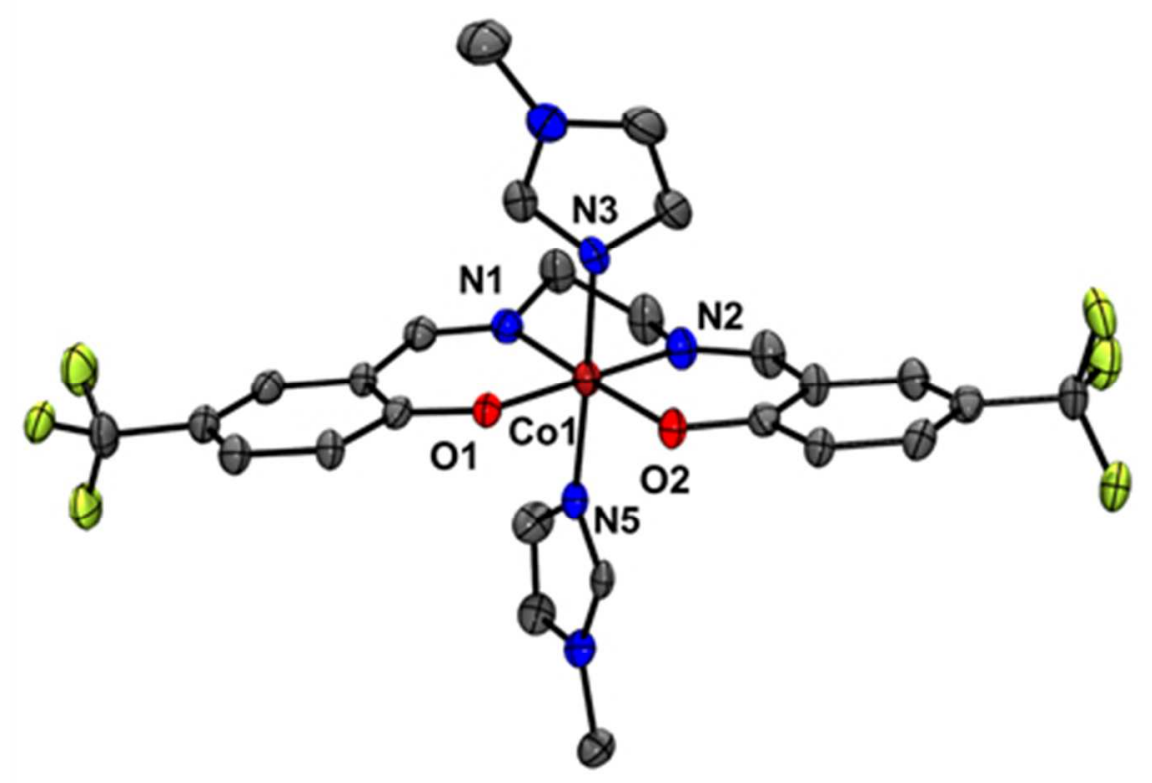

$116 \times 82 \mathrm{~mm}(96 \times 96 \mathrm{DPI})$ 


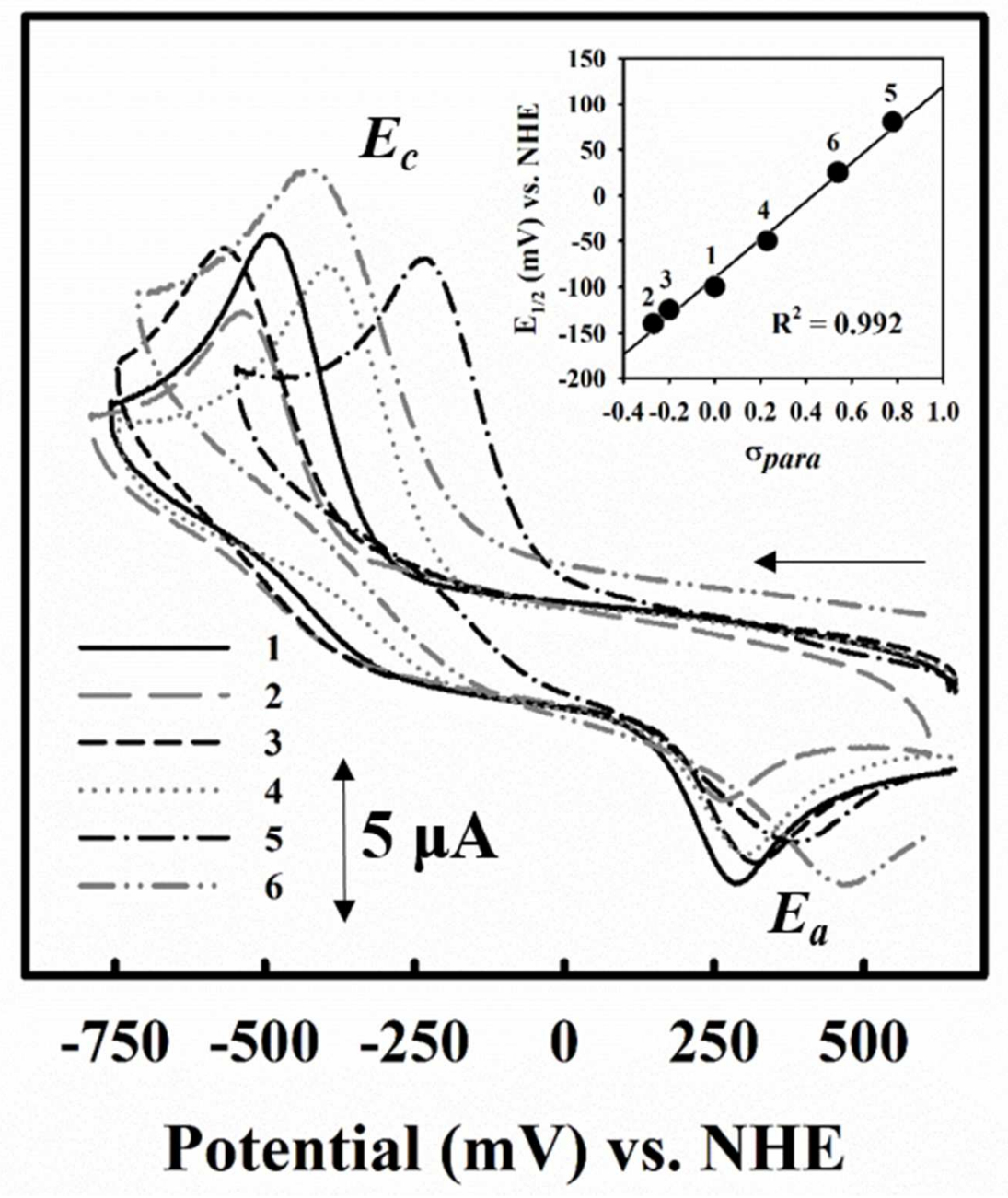

$112 \times 124 \mathrm{~mm}(150 \times 150 \mathrm{DPI})$ 


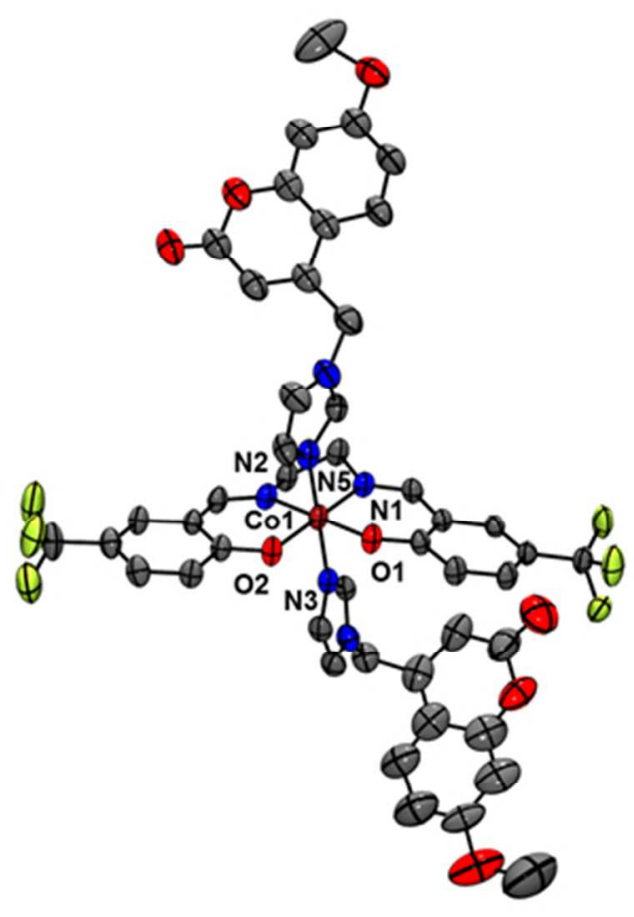

$162 \times 114 \mathrm{~mm}(96 \times 96 \mathrm{DPI})$ 


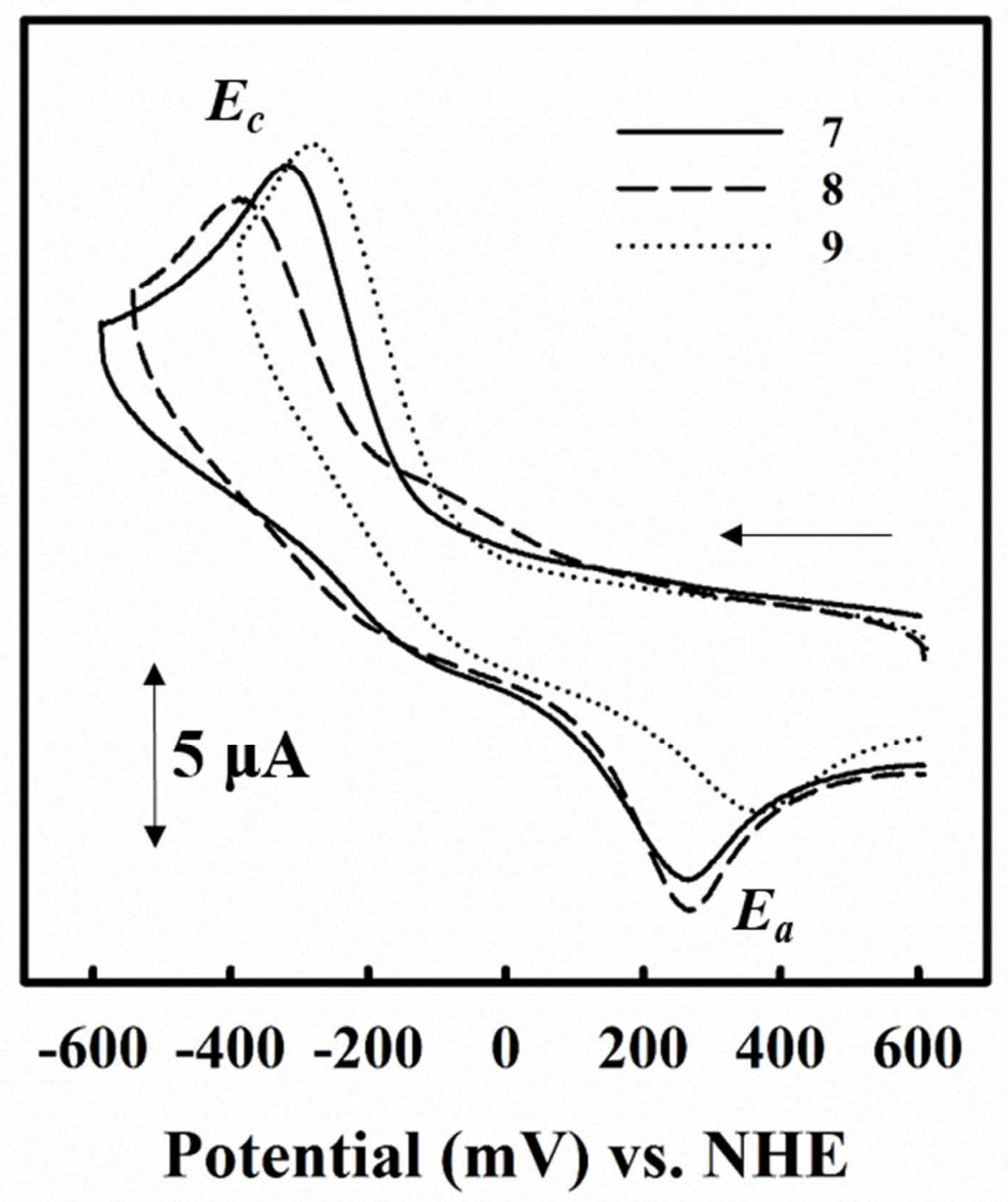

$104 \times 117 \mathrm{~mm}(150 \times 150 \mathrm{DPI})$ 


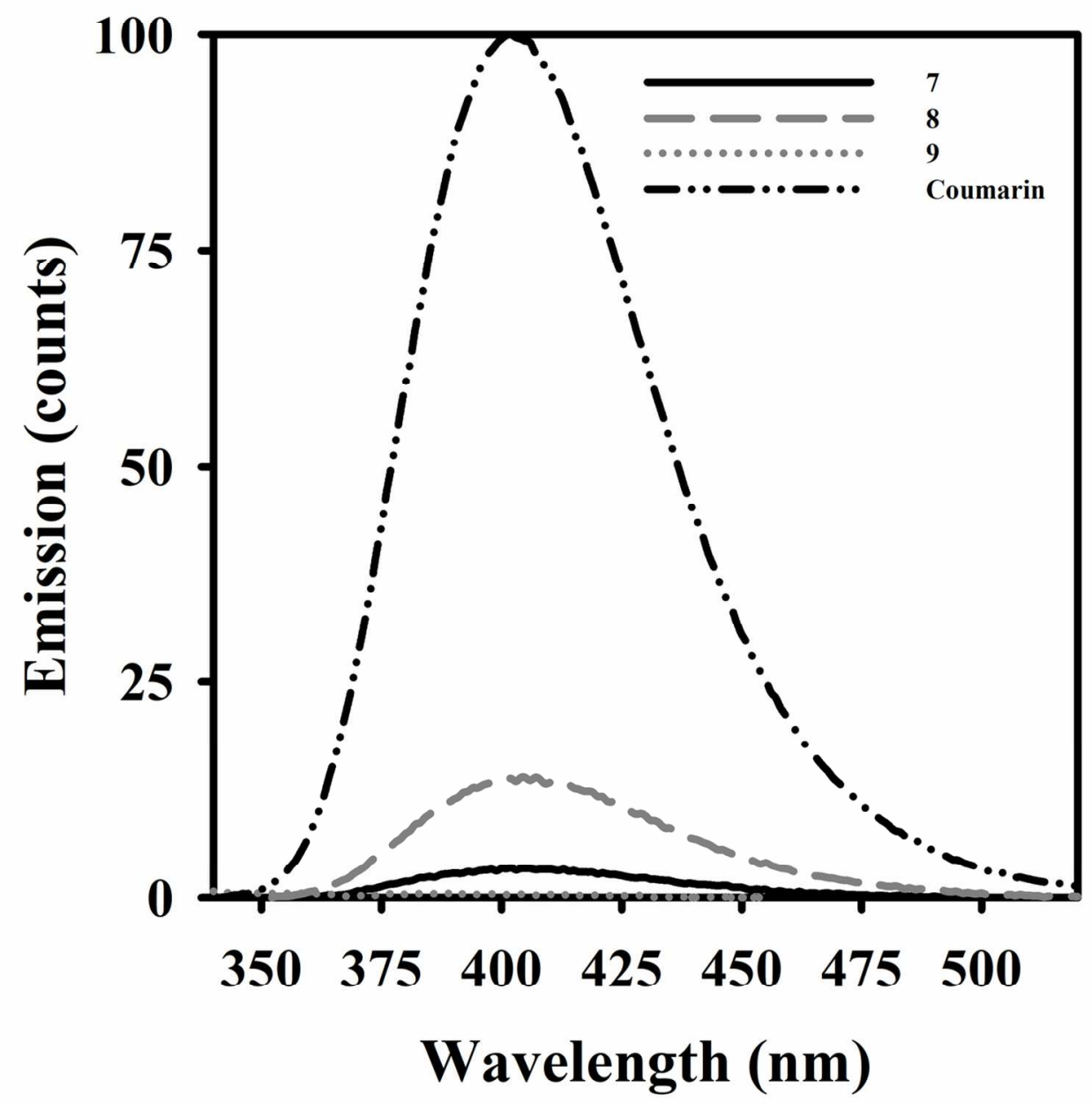

$124 \times 130 \mathrm{~mm}(300 \times 300$ DPI $)$ 


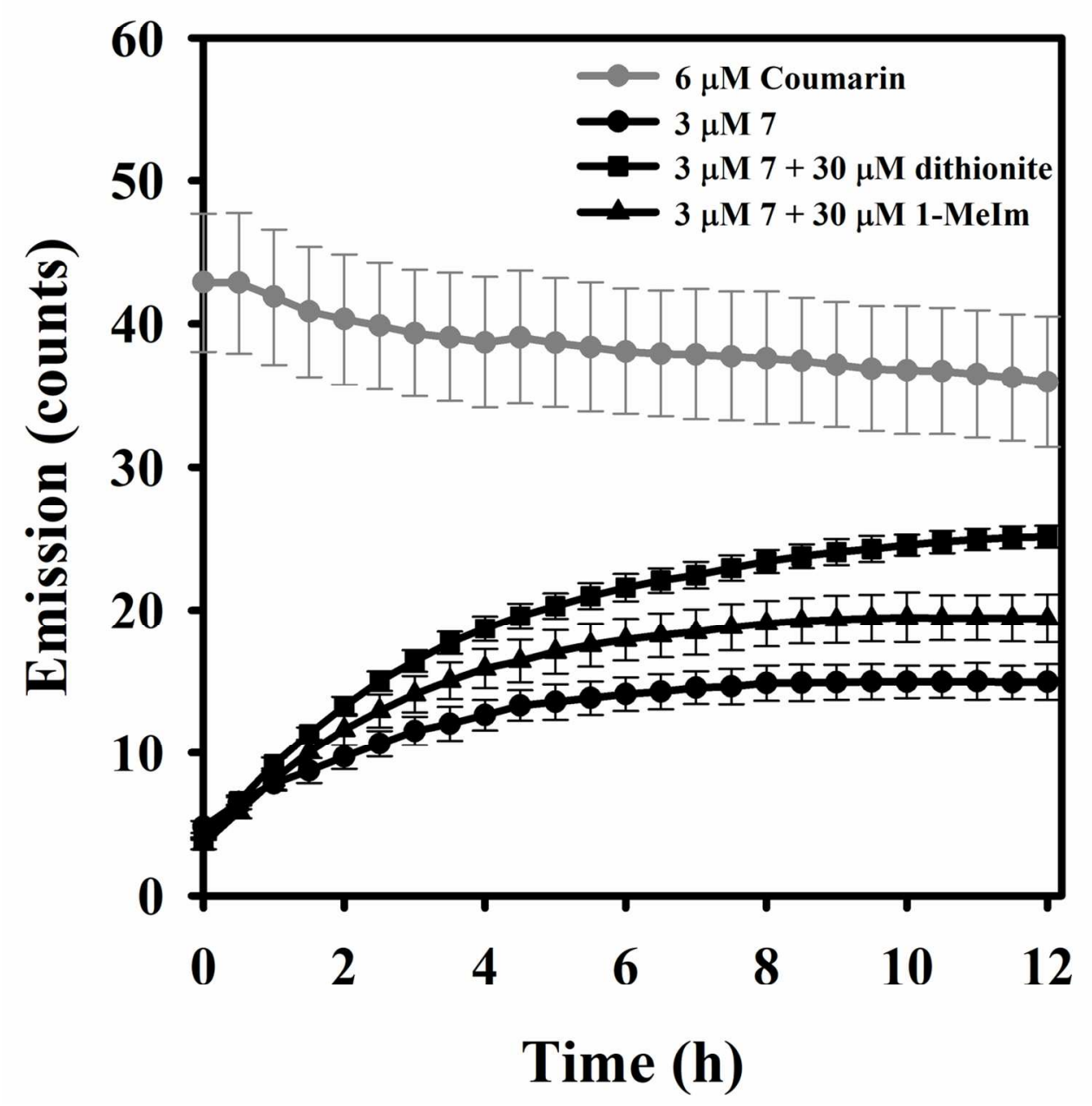

$125 \times 132 \mathrm{~mm}(300 \times 300 \mathrm{DPI})$ 


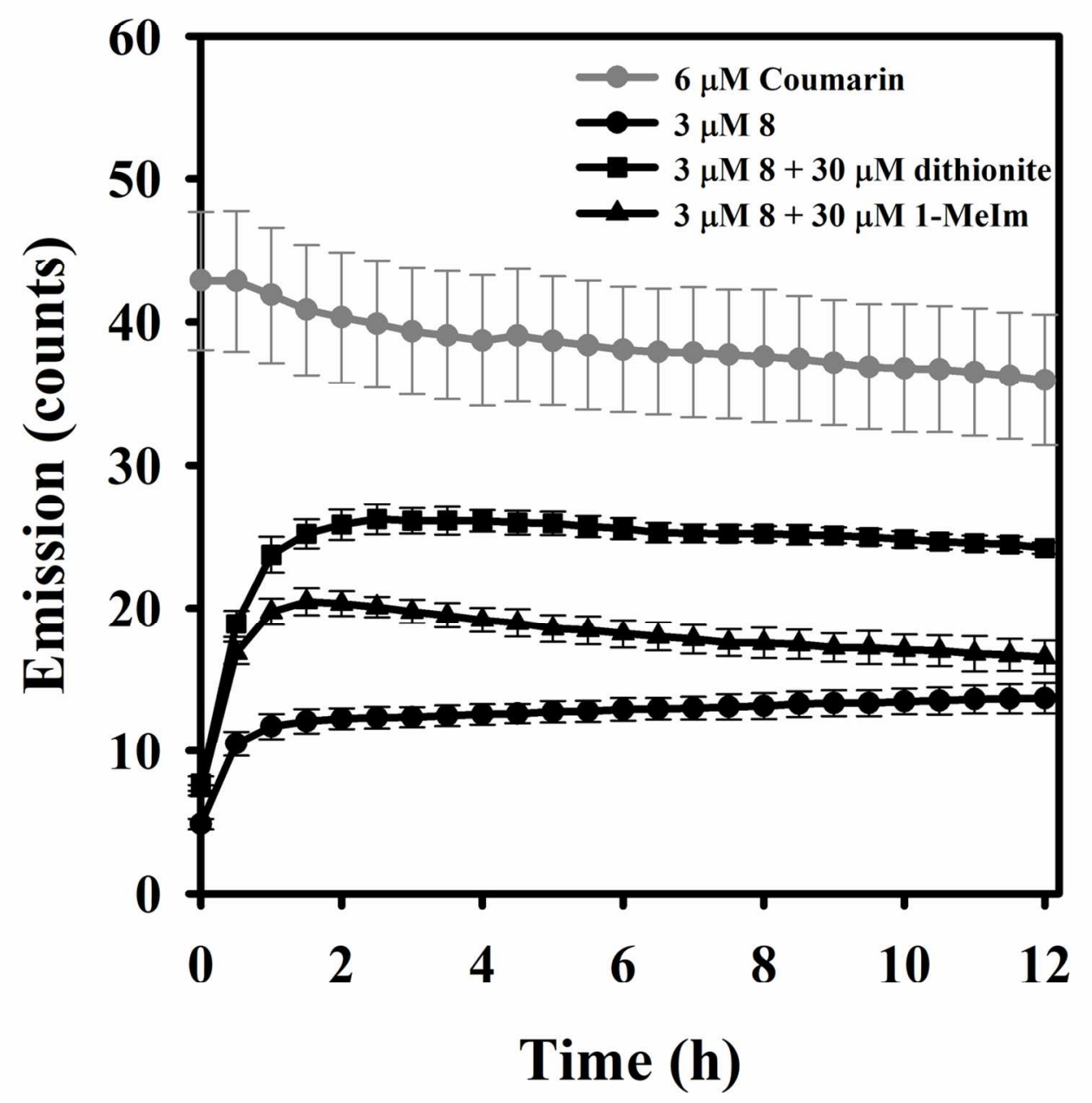

$125 \times 132 \mathrm{~mm}(300 \times 300 \mathrm{DPI})$ 


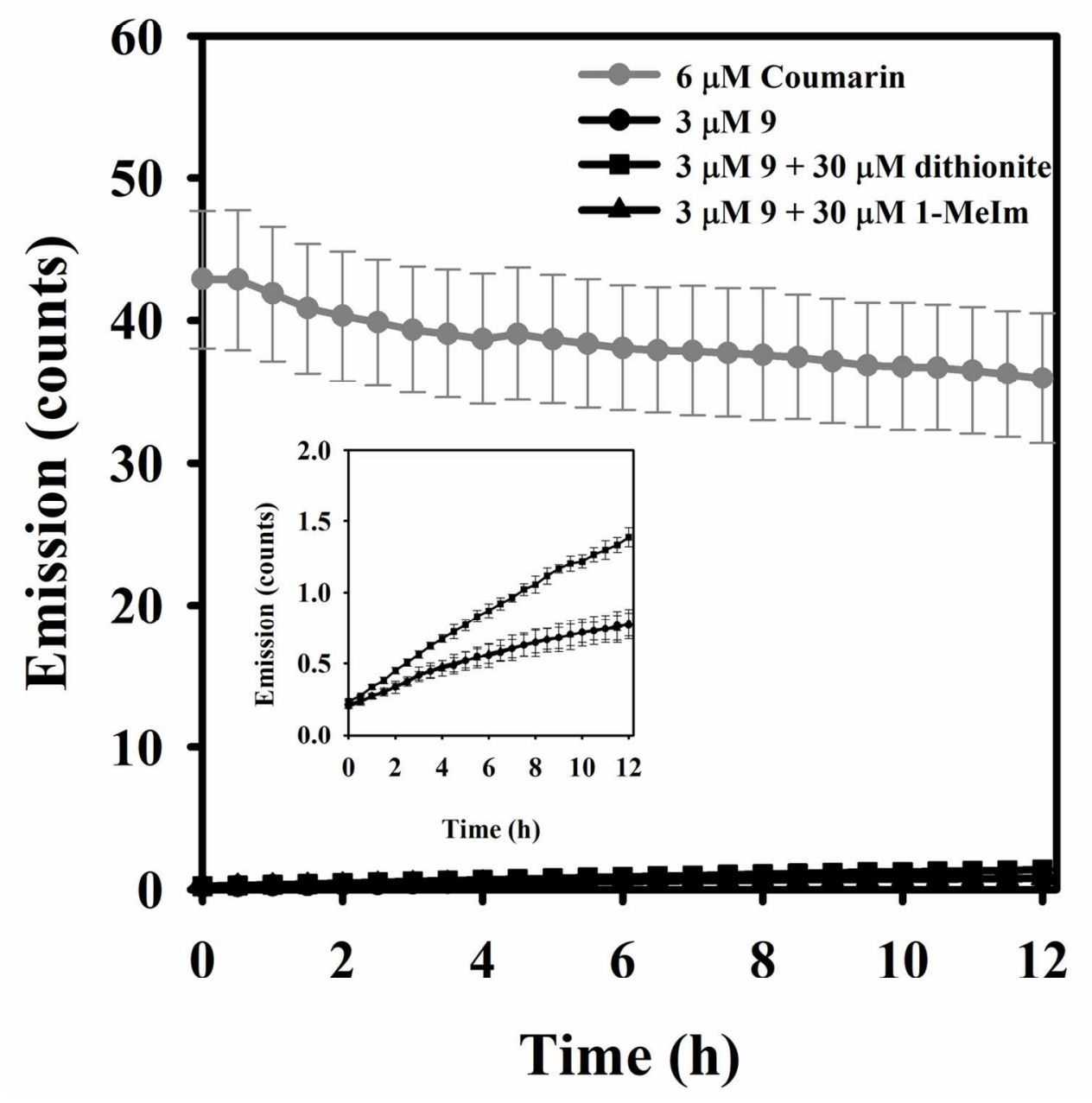

$125 \times 132 \mathrm{~mm}(300 \times 300 \mathrm{DPI})$ 